\title{
Analytical solutions for period-m motions in a periodically forced van der Pol oscillator
}

\author{
Albert C. J. Luo • Arash Baghaei Lakeh
}

Received: 3 April 2013 / Revised: 8 April 2013 / Accepted: 9 April 2013 / Published online: 9 May 2013

(C) Springer-Verlag Berlin Heidelberg 2013

\begin{abstract}
Approximate, analytical solutions of period-m motions in a periodically forced, van der Pol oscillator are obtained through the Fourier series expression, and the corresponding stability and bifurcation analysis of such period-m motions are carried out. To verify the approximate, analytical solutions of period-m motions, numerical simulations are performed, and the numerical results are compared with analytical solutions. The harmonic amplitude distributions are presented to show the significance of harmonic terms in the finite Fourier series expression of the analytical periodic solutions. Period-m motions are separated by quasi-periodic motion or chaos, and the stable period-m motions are in independent periodic motion windows.
\end{abstract}

Keywords Period-m motions - Van der Pol oscillator . Analytical dynamics $\cdot$ Stability and bifurcation .

Harmonic balance method

\section{Introduction}

Consider a periodically forced, van der Pol oscillator

$\ddot{x}+\dot{x}\left(-\alpha_{1}+\alpha_{2} x^{2}\right)+\alpha_{3} x=Q_{0} \cos \Omega t$

where $\alpha_{j}(j=1,2,3)$ are constant parameters. A periodic force is applied to this system with excitation amplitude of $Q_{0}$ and frequency of $\Omega$. In Luo and Lakeh [1], the initial investigation of period- $m$ motions in the van der Pol equation was investigated. Herein a comprehensive investigation of period-m motions will be presented for a better under-

A. C. J. Luo $(\bowtie) \cdot$ A. Baghaei Lakeh

Department of Mechanical and Industrial Engineering, Southern Illinois University Edwardsville, Edwardsville, IL 62026-1805, USA

e-mail: aluo@siue.edu standing of nonlinear characteristics of the van der Pol oscillator entrained by a periodic excitation.

In 1788, Lagrange employed the idea of averaging as a computational technique to discuss periodic motion of the three body problem as a perturbation to the two body problem [2]. In 1920, van der Pol [3] used the same method for periodic motions of self-excited systems in circuits (also see [4]). In 1928, Fatou [5] provided the first proof of asymptotic validity of the method of averaging through the existence of solutions of differential equations. In 1935, Krylov and Bogolyubov [6] developed systematically the method of averaging and the detailed discussion can be found in Bogoliubov and Mitropolsky [7]. Thus, one extensively used the averaging method to determine periodic solutions in nonlinear differential equations. In 1964, Hayashi [8] discussed averaging methods and the principle of harmonic balance, and the Mathieu equation was used to determine the stability of periodic solutions. In 1973, Nayfeh [9] systematically presented the perturbation theory and multi-scale methods, and Nayfeh and Mook [10] applied such perturbation methods for approximate solutions of periodic motions in nonlinear structural vibrations. In 1974, Cap [11] extended an averaging method using Jacobian matrix to determine the perturbation effects of a pendulum oscillator. In 1987, Rand and Armbruster [13] discussed the stability and bifurcation of periodic solutions through the perturbation method. In 1990, Copolla and Rand [12] used the averaging method with elliptic functions for strongly nonlinear oscillators with harmonic excitation. $\mathrm{Xu}$ and Cheung [14] used an averaging technique based on generalized harmonic functions. In 1998, Buonomo $[15,16]$ showed the procedure for periodic solutions of the van der Pol oscillator in power series. Kovacic and Mickens [17] applied the generalized Krylov-Bogoliubov method to the van der Pol oscillator with small nonlinearity for limit cycles. In 2012, Luo [18] developed a generalized harmonic 
balance method to get the approximate analytical solutions of periodic motions and chaos in nonlinear dynamical systems. This method used the finite Fourier series to express periodic motions and the coefficients are slowly time-varying. With averaging, a dynamical system of coefficients are obtained from which the steady-state solution are achieved and the corresponding stability and bifurcation are completed. Luo and Huang [19] used the generalized harmonic balance method to obtain approximate analytical solutions of periodic motions in the Duffing oscillator, and the analytical bifurcation trees of periodic motions to chaos were obtained (also see, Luo and Huang $[20,21])$.

In this paper, period-m motions in a periodically forced, van der Pol oscillator will be discussed through the finite Fourier series expression. The stability and bifurcation analysis of the approximate periodic solutions in the van der Pol oscillator will be discussed from the dynamics of timevarying coefficients in the finite Fourier series solution. Numerical illustrations for period-m motions in the van der Pol oscillator will be completed to demonstrate the approximate periodic solutions.

\section{Analytical solutions}

The standard form of Eq. (1) is

$\ddot{x}+f(x, \dot{x}, t)=0$

where

$f(\dot{x}, x, t)=\dot{x}\left(-\alpha_{1}+\alpha_{2} x^{2}\right)+\alpha_{3} x-Q_{0} \cos \Omega t$.

As in Luo [18], consider the analytical solution of period- $m$ motion, i.e.,

$$
\begin{aligned}
x^{*}(t)= & a_{0}^{(m)}(t)+\sum_{k=1}^{N} b_{k / m}(t) \cos \left(\frac{k}{m} \Omega t\right) \\
& +c_{k / m}(t) \sin \left(\frac{k}{m} \Omega t\right) .
\end{aligned}
$$

Then the first and second order derivatives of $x^{*}(t)$ are

$$
\begin{aligned}
\dot{x}^{*}(t)= & \dot{a}_{0}^{(m)}+\sum_{k=1}^{N}\left(\dot{b}_{k / m}+\frac{k \Omega}{m} c_{k / m}\right) \cos \left(\frac{k \Omega}{m} t\right) \\
& +\left(\dot{c}_{k / m}-\frac{k \Omega}{m} b_{k / m}\right) \sin \left(\frac{k \Omega}{m} t\right), \\
\ddot{x}^{*}(t)= & \ddot{a}_{0}^{(m)}+\sum_{k=1}^{N}\left[\ddot{b}_{k / m}+2 \frac{k \Omega}{m} \dot{c}_{k / m}\right. \\
& \left.-\left(\frac{k \Omega}{m}\right)^{2} b_{k / m}\right] \cos \left(\frac{k \Omega}{m} t\right) \\
& +\left[\ddot{c}_{k / m}-2 \frac{k \Omega}{m} \dot{b}_{k / m}-\left(\frac{k \Omega}{m}\right)^{2} c_{k / m}\right] \sin \left(\frac{k \Omega}{m} t\right) .
\end{aligned}
$$

Substitution of Eqs. (4)-(6) into Eq. (3) and averaging all terms of $\cos (k \Omega t / m)$ and $\sin (k \Omega t / m)$ gives

$$
\begin{aligned}
& \ddot{a}_{0}^{(m)}+F_{0}^{(m)}\left(a_{0}^{(m)}, \mathbf{b}^{(m)}, \mathbf{c}^{(m)}, \dot{a}_{0}^{(m)}, \dot{\mathbf{b}}^{(m)}, \dot{\mathbf{c}}^{(m)}\right)=0 \\
& \ddot{b}_{k / m}+2 \frac{k \Omega}{m} \dot{c}_{k / m}-\left(\frac{k \Omega}{m}\right)^{2} b_{k / m} \\
& \quad+F_{1 k}^{(m)}\left(a_{0}^{(m)}, \mathbf{b}^{(m)}, \mathbf{c}^{(m)}, \dot{a}_{0}^{(m)}, \dot{\mathbf{b}}^{(m)}, \dot{\mathbf{c}}^{(m)}\right)=0 \\
& \ddot{c}_{k / m}-2 \frac{k \Omega}{m} \dot{b}_{k / m}-\left(\frac{k \Omega}{m}\right)^{2} c_{k / m} \\
& \quad+F_{2 k}^{(m)}\left(a_{0}^{(m)}, \mathbf{b}^{(m)}, \mathbf{c}^{(m)}, \dot{a}_{0}^{(m)}, \dot{\mathbf{b}}^{(m)}, \dot{\mathbf{c}}^{(m)}\right)=0 \\
& k=1,2, \ldots, N .
\end{aligned}
$$

The coefficients of constant, $\cos (k \Omega t / m)$ and $\sin (k \Omega t / m)$ for the function $f(x, \dot{x}, t)$ can be obtained in the form of

$$
\begin{aligned}
& F_{0}^{(m)}\left(a_{0}^{(m)}, \mathbf{b}^{(m)}, \mathbf{c}^{(m)}, \dot{a}_{0}^{(m)}, \dot{\mathbf{b}}^{(m)}, \dot{\mathbf{c}}^{(m)}\right) \\
& \quad=-\alpha_{1} \dot{a}_{0}^{(m)}+\alpha_{3} a_{0}^{(m)}+\alpha_{2} f_{0}^{(m)}, \\
& F_{1 k}^{(m)}\left(a_{0}^{(m)}, \mathbf{b}^{(m)}, \mathbf{c}^{(m)}, \dot{a}_{0}^{(m)}, \dot{\mathbf{b}}^{(m)}, \dot{\mathbf{c}}^{(m)}\right) \\
& =-\alpha_{1}\left(\dot{b}_{k / m}+\frac{k}{m} \Omega c_{k / m}\right)+\alpha_{3} b_{k / m}-Q_{0} \delta_{k}^{m}+\alpha_{2} f_{1 k / m}, \\
& F_{2 k}^{(m)}\left(a_{0}^{(m)}, \mathbf{b}^{(m)}, \mathbf{c}^{(m)}, \dot{a}_{0}^{(m)}, \dot{\mathbf{b}}^{(m)}, \dot{\mathbf{c}}^{(m)}\right) \\
& =-\alpha_{1}\left(\dot{c}_{k / m}-\frac{k}{m} \Omega b_{k / m}\right)+\alpha_{3} c_{k / m}+\alpha_{2} f_{2 k / m}
\end{aligned}
$$

where

$$
f_{0}^{(m)}=\dot{a}_{0}^{(m)}\left(a_{0}^{(m)}\right)^{2}+\sum_{s=1}^{6} \sum_{l=1}^{N} \sum_{j=1}^{N} \sum_{i=1}^{N} d_{0 s}^{(m)}(i, j, l)
$$

with

$$
\begin{aligned}
d_{01}^{(m)}(i, j, l)= & \frac{1}{2 N} \dot{a}_{0}^{(m)}\left(b_{i / m} b_{j / m}+c_{i / m} c_{j / m}\right) \delta_{i-j}^{0}, \\
d_{02}^{(m)}(i, j, l)= & \frac{1}{N} a_{0}^{(m)}\left[b_{i / m}\left(\dot{b}_{l / m}+\frac{l}{m} \Omega c_{k / m}\right)\right. \\
& \left.+c_{i / m}\left(\dot{c}_{l / m}-\frac{l}{m} \Omega b_{l / m}\right)\right] \delta_{l-i}^{0}, \\
d_{03}^{(m)}(i, j, l)= & \frac{1}{4}\left(b_{i / m} b_{j / m}-c_{i / m} c_{j / m}\right) \\
& \left(\dot{b}_{l / m}+\frac{l}{m} \Omega c_{l / m}\right) \delta_{l-i-j}^{0}, \\
d_{04}^{(m)}(i, j, l)= & \frac{1}{4}\left(b_{j / m} c_{i / m}+b_{i / m} c_{j / m}\right) \\
& \left(\dot{c}_{l / m}-\frac{l}{m} \Omega b_{l / m}\right) \delta_{l-i-j}^{0}, \\
d_{05}^{(m)}(i, j, l)= & \frac{1}{4}\left(b_{i / m} b_{j / m}+c_{i / m} c_{j / m}\right) \\
& \left(\dot{b}_{l / m}+\frac{l}{m} \Omega c_{l / m}\right)\left(\delta_{l-i+j}^{0}+\delta_{l+i-j}^{0}\right),
\end{aligned}
$$




$$
\begin{aligned}
d_{06}^{(m)}(i, j, l)= & \frac{1}{4}\left(b_{j / m} c_{i / m}-b_{i / m} c_{j / m}\right) \\
& \left(\dot{c}_{l / m}-\frac{l}{m} \Omega b_{l / m}\right)\left(\delta_{l-i+j}^{0}+\delta_{l+i-j}^{0}\right) .
\end{aligned}
$$

The nonlinear term for cosine terms is

$f_{1 k / m}=\sum_{s=1}^{8} \sum_{l=1}^{N} \sum_{j=1}^{N} \sum_{i=1}^{N} d_{1 s}^{(k / m)}(i, j, l)$

with

$$
\begin{aligned}
d_{11}^{(k / m)}(i, j, l)= & \frac{1}{N^{2}}\left[2 \dot{a}_{0}^{(m)} a_{0}^{(m)} b_{i / m} \delta_{i}^{k}\right. \\
& \left.+\frac{1}{N^{2}}\left(a_{0}^{(m)}\right)^{2}\left(\dot{b}_{l / m}+\frac{l}{m} \Omega c_{l / m}\right) \delta_{l}^{k}\right], \\
d_{12}^{(k / m)}(i, j, l)= & \frac{1}{2 N} \dot{a}_{0}^{(m)}\left[\left(b_{i / m} b_{j / m}+c_{i / m} c_{j / m}\right) \delta_{i-j}^{k}\right. \\
& \left.+\left(b_{i / m} b_{j / m}-c_{i / m} c_{j / m}\right) \delta_{i+j}^{k}\right], \\
d_{13}^{(k / m)}(i, j, l)= & \frac{1}{N} a_{0}^{(m)} b_{i / m}\left(\dot{b}_{l / m}+\frac{l}{m} \Omega c_{l / m}\right) \\
& \left(\delta_{|l-i|}^{k}+\delta_{l+i}^{k}\right), \\
d_{14}^{(k / m)}(i, j, l)= & \frac{1}{N} a_{0}^{(m)} c_{i / m}\left(\dot{c}_{l / m}-\frac{l}{m} \Omega b_{l / m}\right) \\
& \left(\delta_{|l-i|}^{k}-\delta_{l+i}^{k}\right), \\
d_{15}^{(k / m)}(i, j, l)= & \frac{1}{4}\left(b_{i / m} b_{j / m}-c_{i / m} c_{j / m}\right) \\
& \left(\dot{b}_{l / m}+\frac{l}{m} \Omega c_{l / m}\right)\left(\delta_{|l-i-j|}^{k}+\delta_{l+i+j}^{k}\right), \\
& \left(\dot{c}_{l / m}-\frac{l}{m} \Omega b_{l / m}\right)\left(\delta_{|l-i-j|}^{k}-\delta_{l+i+j}^{k}\right) . \\
d_{16}^{(k / m)}(i, j, l)= & \frac{1}{4}\left(b_{j / m} c_{i / m}+b_{i / m} c_{j / m}\right) \\
& \left(\dot{c}_{l / m}-\frac{l}{m} \Omega b_{l / m}\right)\left(\delta_{|l-i-j|}^{k}-\delta_{l+i+j}^{k}\right), \\
d_{17}^{(k / m)}(i, j, l)= & \frac{1}{4}\left(b_{j / m} c_{i / m}-b_{i / m} c_{j / m}\right) \\
& \left(\dot{b}_{l / m}+\frac{l}{m} \Omega c_{l / m}\right)\left(\delta_{|l-i-j|}^{k}+\delta_{l+i+j}^{k}\right), \\
& \frac{1}{4}\left(b_{i / m} b_{j / m}+c_{i / m} c_{j / m}\right) \\
& (1, l)
\end{aligned}
$$

The nonlinear term for sine term is

$$
f_{2 k / m}=\sum_{s=1}^{8} \sum_{l=1}^{N} \sum_{j=1}^{N} \sum_{i=1}^{N} d_{2 s}^{(k / m)}(i, j, l)
$$

with

$$
\begin{aligned}
d_{21}^{(k / m)}(i, j, l)= & \frac{1}{N^{2}}\left[2 \dot{a}_{0}^{(m)} a_{0}^{(m)} c_{i / m} \delta_{i}^{k}+\left(a_{0}^{(m)}\right)^{2}\right. \\
& \left.\left(\dot{c}_{k / m}-\frac{l}{m} \Omega b_{l / m}\right) \delta_{l}^{k}\right], \\
d_{22}^{(k / m)}(i, j, l)= & \frac{1}{2 N} \dot{a}_{0}^{(m)}\left[\left(b_{j / m} c_{i / m}-b_{i / m} c_{j / m}\right)\right. \\
& \left.\operatorname{sgn}(i-j) \delta_{|i-j|}^{k}+\left(b_{j / m} c_{i / m}+b_{i / m} c_{j / m}\right) \delta_{i+j}^{k}\right], \\
d_{23}^{(k / m)}(i, j, l)= & \frac{1}{N} a_{0}^{(m)} b_{i / m}\left(\dot{c}_{l / m}-\frac{l}{m} \Omega b_{l / m}\right) \\
& {\left[\delta_{l+i}^{k}+\operatorname{sgn}(l-i) \delta_{|l-i|}^{k}\right], } \\
d_{24}^{(k / m)}(i, j, l)= & \frac{1}{N} a_{0}^{(m)} c_{i / m}\left(\dot{b}_{k / m}+\frac{l}{m} \Omega c_{l / m}\right) \\
& {\left[\delta_{l+i}^{k}-\operatorname{sgn}(l-i) \delta_{|l-i|}^{k}\right], } \\
& \times\left[\operatorname{sgn}(l-i+j) \delta_{|l-i+j|}^{k}-\operatorname{sgn}(l+i-j) \delta_{|l+i-j|}^{k}\right] \\
& \times\left[\delta_{l+i+j}^{k}-\operatorname{sgn}(l-i-j) \delta_{|l-i-j|}^{k}\right] \\
d_{25}^{(k / m)}(i, j, l) & \left(b_{i / m} c_{j / m}+b_{j / m} c_{i / m}\right)\left(\dot{b}_{l / m}+\frac{l}{m} \Omega c_{l / m}\right) \\
& \times\left[\delta_{l+i+j}^{k}+\operatorname{sgn}(l-i-j) \delta_{|l-i-j|}^{k}\right], \\
d_{28}^{(k / m)}(i, j, l)= & \frac{1}{4}\left(b_{i / m} c_{j / m}-b_{j / m} c_{i / m}\right)\left(\dot{b}_{l / m}+\frac{l}{m} \Omega c_{l / m}\right) \\
d_{26}^{(k / m)}(i, j, l)= & \frac{1}{4}\left(b_{i / m} b_{j / m}+c_{i / m} c_{j / m}\right)\left(\dot{c}_{l / m}-\frac{l}{m} \Omega b_{l / m}\right) \\
& \\
& \\
& \\
& \\
&
\end{aligned}
$$

Define new vectors

$$
\begin{aligned}
\mathbf{z}^{(m)} & \triangleq\left(a_{0}^{(m)}, \mathbf{b}^{(m)}, \mathbf{c}^{(m)}\right)^{\mathrm{T}} \\
& =\left(a_{0}^{(m)}, b_{1 / m}, \ldots, b_{N / m}, c_{1 / m}, \ldots, c_{N / m}\right)^{\mathrm{T}} \\
& \equiv\left(z_{0}^{(m)}, z_{1}^{(m)}, \ldots, z_{2 N}^{(m)}\right)^{\mathrm{T}} \\
\mathbf{z}_{1}^{(m)} & =\dot{\mathbf{z}}^{(m)}=\left(\dot{a}_{0}^{(m)}, \dot{\mathbf{b}}^{(m)}, \dot{\mathbf{c}}^{(m)}\right)^{\mathrm{T}} \\
& =\left(\dot{a}_{0}^{(m)}, \dot{b}_{1 / m}, \ldots, \dot{b}_{N / m}, \dot{c}_{1 / m}, \ldots, \dot{c}_{N / m}\right)^{\mathrm{T}} \\
& \equiv\left(\dot{z}_{0}^{(m)}, \dot{z}_{1}^{(m)}, \ldots, \dot{z}_{2 N}^{(m)}\right)^{\mathrm{T}}
\end{aligned}
$$

where

$\mathbf{b}^{(m)}=\left(b_{1 / m}, b_{2 / m}, \ldots, b_{N / m}\right)^{\mathrm{T}}$,
$\mathbf{c}^{(m)}=\left(c_{1 / m}, c_{2 / m}, \ldots, c_{N / m}\right)^{\mathrm{T}}$.

Equation (7) becomes

$\dot{\mathbf{z}}^{(m)}=\mathbf{z}_{1}^{(m)}$ and $\dot{\mathbf{z}}_{1}^{(m)}=\mathbf{g}^{(m)}\left(\mathbf{z}^{(m)}, \mathbf{z}_{1}^{(m)}\right)$ 
where

$$
\begin{aligned}
\mathbf{g}^{(m)} & \left(\mathbf{z}^{(m)}, \mathbf{z}_{1}^{(m)}\right) \\
= & \left(\begin{array}{c}
-F_{0}^{(m)}\left(\mathbf{z}^{(m)}, \mathbf{z}_{1}^{(m)}\right) \\
-\mathbf{F}_{1}^{(m)}\left(\mathbf{z}^{(m)}, \mathbf{z}_{1}^{(m)}\right)-2 \mathbf{k}_{1} \frac{\Omega}{m} \dot{\mathbf{b}}^{(m)}+\mathbf{k}_{2}\left(\frac{\Omega}{m}\right)^{2} \mathbf{b}^{(m)} \\
-\mathbf{F}_{2}^{(m)}\left(\mathbf{z}^{(m)}, \mathbf{z}_{1}^{(m)}\right)+2 \mathbf{k}_{1} \frac{\Omega}{m} \dot{\mathbf{c}}^{(m)}+\mathbf{k}_{2}\left(\frac{\Omega}{m}\right)^{2} \mathbf{c}^{(m)}
\end{array}\right)
\end{aligned}
$$

where

$\mathbf{k}_{1}=\operatorname{diag}(1,2, \ldots, N)$,

$\mathbf{k}_{2}=\operatorname{diag}\left(1,2^{2}, \ldots, N^{2}\right)$;

$\mathbf{F}_{1}^{(m)}=\left(F_{11}^{(m)}, F_{12}^{(m)}, \ldots, F_{1 N}^{(m)}\right)^{\mathrm{T}}$,

$\mathbf{F}_{2}^{(m)}=\left(F_{21}^{(m)}, F_{22}^{(m)}, \ldots, F_{2 N}^{(m)}\right)^{\mathrm{T}}$

for $N=1,2, \ldots, \infty$;

and

$\mathbf{y}^{(m)} \equiv\left(\mathbf{z}^{(m)}, \mathbf{z}_{1}^{(m)}\right)$ and $\mathbf{f}^{(m)}=\left(\mathbf{z}_{1}^{(m)}, \mathbf{g}^{(m)}\right)^{\mathrm{T}}$

Thus, Eq. (17) becomes

$\dot{\mathbf{y}}^{(m)}=\mathbf{f}^{(m)}\left(\mathbf{y}^{(m)}\right)$

The steady-state solutions for periodic motion can be obtained by setting $\dot{\mathbf{y}}^{(m)}=\mathbf{0}$, i.e.,

$F_{0}^{(m)}\left(a_{0}^{(m) *}, \mathbf{b}^{(m) *}, \mathbf{c}^{(m) *}, 0, \mathbf{0}, \mathbf{0}\right)=0$,

$\mathbf{F}_{1}^{(m)}\left(a_{0}^{(m) *}, \mathbf{b}^{(m) *}, \mathbf{c}^{(m) *}, 0, \mathbf{0}, \mathbf{0}\right)-\frac{\Omega^{2}}{m^{2}} \mathbf{k}_{2} \mathbf{b}^{(m) *}=\mathbf{0}$,

$\mathbf{F}_{2}^{(m)}\left(a_{0}^{(m) *}, \mathbf{b}^{(m) *}, \mathbf{c}^{(m) *}, 0, \mathbf{0}, \mathbf{0}\right)-\frac{\Omega^{2}}{m^{2}} \mathbf{k}_{2} \mathbf{c}^{(m) *}=\mathbf{0}$.

The $(2 N+1)$ nonlinear equations in Eq. (22) are solved by the Newton-Raphson method. In Luo [18], the linearized equation at $\mathbf{y}^{(m) *}=\left(\mathbf{z}^{(m) *}, \mathbf{0}\right)^{\mathrm{T}}$ is

$\Delta \dot{\mathbf{y}}^{(m)}=D \mathbf{f}^{(m)}\left(\mathbf{y}^{*(m)}\right) \Delta \mathbf{y}^{(m)}$

where

$D \mathbf{f}^{(m)}\left(\mathbf{y}^{*(m)}\right)=\partial \mathbf{f}^{(m)}\left(\mathbf{y}^{(m)}\right) /\left.\partial \mathbf{y}^{(m)}\right|_{\mathbf{y}^{(m) *}}$

The corresponding eigenvalues are determined by

$$
\left|D \mathbf{f}^{(m)}\left(\mathbf{y}^{*(m)}\right)-\lambda \mathbf{I}_{2(2 N+1) \times 2(2 N+1)}\right|=0 .
$$

where

$$
D \mathbf{f}\left(\mathbf{y}^{(m)^{*}}\right)=\left[\begin{array}{ll}
\mathbf{0}_{(2 N+1) \times(2 N+1)} & \mathbf{I}_{(2 N+1) \times(2 N+1)} \\
\mathbf{G}_{(2 N+1) \times(2 N+1)} & \mathbf{H}_{(2 N+1) \times(2 N+1)}
\end{array}\right]
$$

and

$$
\begin{aligned}
& \mathbf{G}=\frac{\partial \mathbf{g}^{(m)}}{\partial \mathbf{z}^{(m)}}=\left(\mathbf{G}^{(0)}, \mathbf{G}^{(c)}, \mathbf{G}^{(s)}\right)^{\mathrm{T}} \\
& \mathbf{G}^{(0)}=\left(G_{0}^{(0)}, G_{1}^{(0)}, \ldots, G_{2 N}^{(0)}\right), \\
& \mathbf{G}^{(c)}=\left(\mathbf{G}_{1}^{(c)}, \mathbf{G}_{2}^{(c)}, \ldots, \mathbf{G}_{N}^{(c)}\right)^{\mathrm{T}} \\
& \mathbf{G}^{(s)}=\left(\mathbf{G}_{1}^{(s)}, \mathbf{G}_{2}^{(s)}, \ldots, \mathbf{G}_{N}^{(s)}\right)^{\mathrm{T}}
\end{aligned}
$$

for $N=1,2, \ldots \infty$ with

$\mathbf{G}_{k}^{(c)}=\left(G_{k 0}^{(c)}, G_{k 1}^{(c)}, \ldots, G_{k(2 N)}^{(c)}\right)$,

$\mathbf{G}_{k}^{(s)}=\left(G_{k 0}^{(s)}, G_{k 1}^{(s)}, \ldots, G_{k(2 N)}^{(s)}\right)$

for $k=1,2, \ldots N$. The corresponding components are

$G_{r}^{(0)}=-\alpha_{3} \delta_{0}^{r}-\alpha_{2} g_{r}^{(0)}$,

$G_{k r}^{(c)}=\left(\frac{k \Omega}{m}\right)^{2} \delta_{k}^{r}+\alpha_{1}\left(\frac{k}{m} \Omega\right) \delta_{k+N}^{r}-\alpha_{3} \delta_{k}^{r}-\alpha_{2} g_{k r}^{(c)}$,

$G_{k r}^{(s)}=\left(\frac{k \Omega}{m}\right)^{2} \delta_{k+N}^{r}-\alpha_{1}\left(\frac{k}{m} \Omega\right) \delta_{k}^{r}-\alpha_{3} \delta_{k+N}^{r}-\alpha_{2} g_{k r}^{(s)}$

where

$g_{r}^{(0)}=g_{0}^{(0)}(r)+\sum_{n=1}^{16} \sum_{l=1}^{N} \sum_{j=1}^{N} \sum_{i=1}^{N} g_{n}^{(0)}(i, j, l, r)$

with

$$
\begin{aligned}
& g_{0}^{(0)}(r)=2 \dot{a}_{0}^{(m)} a_{0}^{(m)} \delta_{r}^{0} \\
& g_{1}^{(0)}(i, j, l, r)=\frac{1}{N} b_{i / m}\left(\dot{b}_{k / m}+\frac{l}{m} \Omega c_{l / m}\right) \delta_{l-i}^{0} \delta_{0}^{r} \\
& g_{2}^{(0)}(i, j, l, r)=\frac{1}{N} c_{i / m}\left(\dot{c}_{l / m}-\frac{l}{m} \Omega b_{l / m}\right) \delta_{l-i}^{0} \delta_{0}^{r} \\
& g_{0}^{(0)}(r)=2 \dot{a}_{0}^{(m)} a_{0}^{(m)} \delta_{r}^{0} \\
& g_{3}^{(0)}(i, j, l, r)=\frac{1}{N}\left[\dot{a}_{0}^{(m)} b_{i / m} \delta_{i-j}^{0}+a_{0}^{(m)}\right. \\
& \left.\left(\dot{b}_{k / m}+\frac{l}{m} \Omega c_{l / m}\right) \delta_{l-i}^{0}\right] \delta_{i}^{r} \\
& g_{4}^{(0)}(i, j, l, r)=-\frac{1}{N} a_{0}^{(m)} \frac{k}{m} \Omega c_{i / m} \delta_{l-i}^{0} \delta_{l}^{r} \\
& g_{5}^{(0)}(i, j, l, r)=\frac{1}{2} b_{j / m}\left(\dot{b}_{l / m}+\frac{l}{m} \Omega c_{l / m}\right) \delta_{l-i-j}^{0} \delta_{i}^{r} \\
& g_{6}^{(0)}(i, j, l, r)=\frac{1}{2} c_{j / m}\left(\dot{c}_{l / m}-\frac{l}{m} \Omega b_{l / m}\right) \delta_{l-i-j}^{0} \delta_{i}^{r} \\
& g_{7}^{(0)}(i, j, l, r)=-\frac{1}{4} \frac{l}{m} \Omega\left(b_{j / m} c_{i / m}+b_{i / m} c_{j / m}\right) \delta_{l-i-j}^{0} \delta_{l}^{r} \\
& g_{8}^{(0)}(i, j, l, r)=\frac{1}{2} b_{j / m}\left(\dot{b}_{l / m}+\frac{l}{m} \Omega c_{l / m}\right)\left(\delta_{l-i+j}^{0}+\delta_{l+i-j}^{0}\right) \delta_{i}^{r} \\
& g_{9}^{(0)}(i, j, l, r)=-\frac{1}{4} \frac{l}{m} \Omega\left(b_{j / m} c_{i / m}-b_{i / m} c_{j / m}\right) \\
& \left(\delta_{l-i+j}^{0}+\delta_{l+i-j}^{0}\right) \delta_{l}^{r}
\end{aligned}
$$


$g_{10}^{(0)}(i, j, l, r)=\frac{1}{N}\left[\dot{a}_{0}^{(m)} c_{j / m} \delta_{i-j}^{0} \delta_{j+N}^{r}+a_{0}^{(m)} \frac{l}{m} \Omega b_{i / m} \delta_{l-i}^{0} \delta_{l+N}^{r}\right]$

$g_{11}^{(0)}(i, j, l, r)=\frac{1}{N} a_{0}^{(m)}\left(\dot{c}_{l / m}-\frac{l}{m} \Omega b_{l / m}\right) \delta_{l-i}^{0} \delta_{i+N}^{r}$

$g_{12}^{(0)}(i, j, l, r)=-\frac{1}{2} c_{j / m}\left(\dot{b}_{l / m}+\frac{l}{m} \Omega c_{l / m}\right) \delta_{l-i-j}^{0} \delta_{i+N}^{r}$

$g_{13}^{(0)}(i, j, l, r)=\frac{1}{4} \frac{l}{m} \Omega\left(b_{i / m} b_{j / m}-c_{i / m} c_{j / m}\right) \delta_{l-i-j}^{0} \delta_{l+N}^{r}$

$g_{14}^{(0)}(i, j, l, r)=\frac{1}{2} b_{j / m}\left(\dot{c}_{l / m}-\frac{l}{m} \Omega b_{l / m}\right) \delta_{l-i-j}^{0} \delta_{i+N}^{r}$

$g_{15}^{(0)}(i, j, l, r)=\frac{1}{2} c_{j / m}\left(\dot{b}_{l / m}+\frac{l}{m} \Omega c_{l / m}\right)$

$$
\left(\delta_{l-i+j}^{0}+\delta_{l+i-j}^{0}\right) \delta_{i+N}^{r}
$$

$g_{16}^{(0)}(i, j, l, r)=\frac{1}{4} \frac{l}{m} \Omega\left(b_{i / m} b_{j / m}+c_{i / m} c_{j / m}\right)$

$$
\left(\delta_{l-i+j}^{0}+\delta_{l+i-j}^{0}\right) \delta_{l+N}^{r}
$$

and

$g_{k r}^{(c)}=\sum_{n=1}^{18} \sum_{l=1}^{N} \sum_{j=1}^{N} \sum_{i=1}^{N} g_{n}^{(1)}(i, j, l, k, r)$

with

$$
\begin{aligned}
g_{1}^{(1)}(i, j, l, k, r)= & \frac{2}{N^{2}}\left[\dot{a}_{0}^{(m)} b_{i / m} \delta_{i}^{k}+a_{0}^{(m)}\right. \\
& \left.\left(\dot{b}_{l / m}+\frac{l}{m} \Omega c_{l / m}\right) \delta_{l}^{k}\right] \delta_{0}^{r} \\
g_{2}^{(1)}(i, j, l, k, r)= & \frac{1}{N} b_{i / m}\left(\dot{b}_{l / m}+\frac{l}{m} \Omega c_{l / m}\right) \\
& \left(\delta_{|l-i|}^{k}+\delta_{l+i}^{k}\right) \delta_{0}^{r} \\
g_{3}^{(1)}(i, j, l, k, r)= & \frac{1}{N} c_{i / m}\left(\dot{c}_{k / m}-\frac{l}{m} \Omega b_{l / m}\right) \\
& \left(\delta_{|l-i|}^{k}-\delta_{l+i}^{k}\right) \delta_{0}^{r}
\end{aligned}
$$$$
g_{4}^{(1)}(i, j, l, k, r)=\frac{2}{N^{2}} \dot{a}_{0}^{(m)} a_{0}^{(m)} \delta_{i}^{k} \delta_{i}^{r}+\frac{1}{N} \dot{a}_{0}^{(m)} b_{j / m} \delta_{i}^{r}
$$$$
\left(\delta_{|i-j|}^{k}+\delta_{i+j}^{k}\right) \delta_{i}^{r}
$$$$
g_{5}^{(1)}(i, j, l, k, r)=\frac{1}{N} a_{0}^{(m)}\left(\dot{b}_{l / m}+\frac{l}{m} \Omega c_{l / m}\right)
$$$$
\left(\delta_{|l-i|}^{k}+\delta_{l+i}^{k}\right) \delta_{i}^{r}
$$$$
g_{6}^{(1)}(i, j, l, k, r)=-\frac{1}{N} a_{0}^{(m)} \frac{l}{m} \Omega c_{i / m}\left(\delta_{|l-i|}^{k}-\delta_{l+i}^{k}\right) \delta_{k}^{r}
$$$$
g_{7}^{(1)}(i, j, l, k, r)=\frac{1}{2} b_{j / m}\left(\dot{b}_{l / m}+\frac{l}{m} \Omega c_{l / m}\right)
$$

$$
\left(\delta_{|l-i-j|}^{k}+\delta_{l+i+j}^{k}\right) \delta_{i}^{r}
$$

$g_{8}^{(1)}(i, j, l, k, r)=\frac{1}{2} c_{j / m}\left(\dot{c}_{l / m}-\frac{l}{m} \Omega b_{l / m}\right)$

$$
\left(\delta_{|l-i-j|}^{k}-\delta_{l+i+j}^{k}\right) \delta_{i}^{r}
$$

$g_{9}^{(1)}(i, j, l, k, r)=-\frac{1}{4} \frac{l}{m} \Omega\left(b_{j / m} c_{i / m}+b_{i / m} c_{j / m}\right)$

$$
\left(\delta_{|l-i-j|}^{k}-\delta_{l+i+j}^{k}\right) \delta_{l}^{r}
$$

$g_{10}^{(1)}(i, j, l, k, r)=\frac{1}{2} b_{j / m}\left(\dot{b}_{l / m}+\frac{l}{m} \Omega c_{l / m}\right)$

$$
\left(\delta_{|l-i+j|}^{k}+\delta_{|l+i-j|}^{k}\right) \delta_{i}^{r}
$$

$g_{11}^{(1)}(i, j, l, k, r)=-\frac{1}{4} \frac{l}{m} \Omega\left(b_{j / m} c_{i / m}-b_{i / m} c_{j / m}\right)$

$$
\left(\delta_{|l-i+j|}^{k}-\delta_{|l+i-j|}^{k}\right) \delta_{l}^{r}
$$

$g_{12}^{(1)}(i, j, l, k, r)=\frac{1}{N^{2}} \frac{l}{m} \Omega a_{0}^{(m)} \delta_{l}^{k} \delta_{l+N}^{r}+\frac{1}{N} \dot{a}_{0}^{(m)} c_{j / m}$

$$
\left(\delta_{|i-j|}^{k}-\delta_{i+j}^{k}\right) \delta_{i+N}^{r}
$$

$g_{13}^{(1)}(i, j, l, k, r)=\frac{1}{N} \frac{k}{m} \Omega a_{0}^{(m)} b_{i / m}\left(\delta_{|l-i|}^{k}+\delta_{l+i}^{k}\right) \delta_{l+N}^{r}$

$g_{14}^{(1)}(i, j, l, k, r)=-\frac{1}{2} c_{j}\left(\dot{b}_{l / m}+\frac{l}{m} \Omega c_{l / m}\right)$

$$
\left(\delta_{|l-i-j|}^{k}+\delta_{l+i+j}^{k}\right) \delta_{i+N}^{r}
$$

$g_{15}^{(1)}(i, j, l, k, r)=\frac{1}{4} \frac{l}{m} \Omega\left(b_{i / m} b_{j / m}-c_{i / m} c_{j / m}\right)$

$$
\left(\delta_{|l-i-j|}^{k}+\delta_{l+i+j}^{k}\right) \delta_{l+N}^{r}
$$

$g_{16}^{(1)}(i, j, l, k, r)=\frac{1}{2} b_{j / m}\left(\dot{c}_{l / m}-\frac{l}{m} \Omega b_{l / m}\right)$

$$
\left(\delta_{|l-i-j|}^{k}-\delta_{l+i+j}^{k}\right) \delta_{i+N}^{r}
$$

$g_{17}^{(1)}(i, j, l, k, r)=\frac{1}{2} c_{j / m}\left(\dot{b}_{l / m}+\frac{l}{m} \Omega c_{l / m}\right)$

$$
\left(\delta_{|l-i+j|}^{k}+\delta_{|l+i-j|}^{k}\right) \delta_{i+N}^{r}
$$

$g_{18}^{(1)}(i, j, l, k, r)=\frac{1}{4} \frac{l}{m} \Omega\left(b_{i / m} b_{j / m}+c_{i / m} c_{j / m}\right)$

$$
\left(\delta_{|l-i+j|}^{k}+\delta_{|l+i-j|}^{k}\right) \delta_{l+N}^{r}
$$

and

$g_{k r}^{(s)}=\sum_{n=1}^{21} \sum_{l=1}^{N} \sum_{j=1}^{N} \sum_{i=1}^{N} g_{n}^{(2)}(i, j, l, k, r)$

with

$$
\begin{aligned}
g_{1}^{(2)}(i, j, l, k, r)= & \frac{2}{N^{2}}\left[\dot{a}_{0}^{(m)} c_{i / m} \delta_{i}^{k}+a_{0}^{(m)}\right. \\
& \left.\left(\dot{c}_{l / m}-\frac{l}{m} \Omega b_{l / m}\right) \delta_{l}^{k}\right] \delta_{0}^{r} \\
g_{1}^{(2)}(i, j, l, k, r)= & 2 \frac{\dot{a}_{0}}{N^{2}} c_{i / m} \delta_{i}^{k} \delta_{0}^{r} \\
& +2 \frac{a_{0}}{N^{2}}\left(\dot{c}_{l / m}-\frac{l}{m} \Omega b_{l / m}\right) \delta_{l}^{k} \delta_{0}^{r} \\
g_{2}^{(2)}(i, j, l, k, r)= & \frac{1}{N} b_{i / m}\left(\dot{c}_{l / m}-\frac{l}{m} \Omega b_{l / m}\right)
\end{aligned}
$$




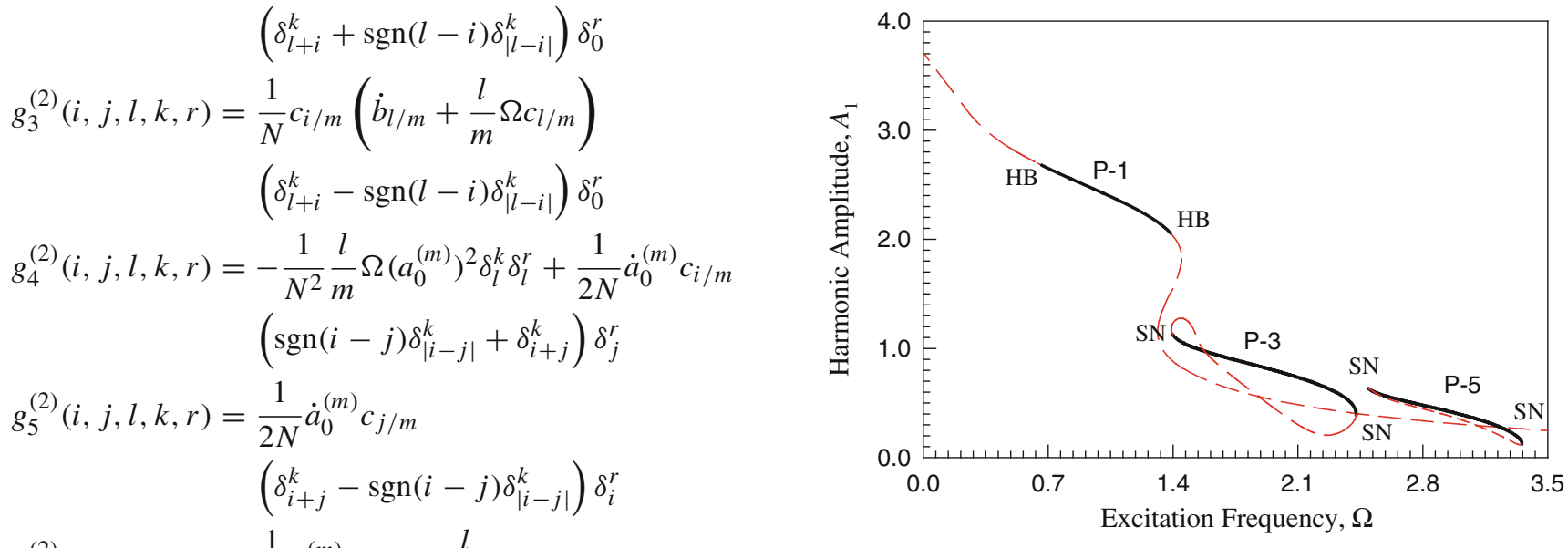

$g_{6}^{(2)}(i, j, l, k, r)=\frac{1}{N} a_{0}^{(m)}\left(\dot{c}_{l / m}-\frac{l}{m} \Omega b_{l / m}\right)$$$
\left(\delta_{l+i}^{k}+\operatorname{sgn}(l-i) \delta_{|l-i|}^{k}\right) \delta_{i}^{r}
$$

$g_{7}^{(2)}(i, j, l, k, r)=-\frac{1}{N} \frac{l}{m} \Omega a_{0}^{(m)} b_{i / m}$

$\left[\delta_{l+i}^{k}+\operatorname{sgn}(l-i) \delta_{|l-i|}^{k}\right] \delta_{l}^{r}$

$g_{8}^{(2)}(i, j, l, k, r)=\frac{1}{2} c_{j / m}\left(\dot{b}_{l / m}+\frac{l}{m} \Omega c_{l / m}\right)$

$\left(\delta_{l+i+j}^{k}-\operatorname{sgn}(l-i-j) \delta_{|l-i-j|}^{k}\right] \delta_{i}^{r}$

$g_{9}^{(2)}(i, j, l, k, r)=\frac{1}{2} b_{j / m}\left(\dot{c}_{l / m}-\frac{l}{m} \Omega b_{l / m}\right)$

$\left[\delta_{l+i+j}^{k}+\operatorname{sgn}(l-i-j) \delta_{|l-i-j|}^{k}\right] \delta_{i}^{r}$

$g_{10}^{(2)}(i, j, l, k, r)=-\frac{1}{4} \frac{l}{m} \Omega\left(b_{i / m} b_{j / m}-c_{i / m} c_{j / m}\right)$

$\left[\delta_{l+i+j}^{k}+\operatorname{sgn}(l-i-j) \delta_{|l-i-j|}^{k}\right] \delta_{l}^{r}$

$g_{11}^{(2)}(i, j, l, k, r)=\frac{1}{4} b_{j / m}\left(\dot{c}_{l / m}-\frac{l}{m} \Omega b_{l / m}\right)$

$\left[\operatorname{sgn}(l-i+j) \delta_{|l-i+j|}^{k}\right.$

$\left.+\operatorname{sgn}(l+i-j) \delta_{|l+i-j|}^{k}\right] \delta_{i}^{r}$

$g_{12}^{(2)}(i, j, l, k, r)=-\frac{1}{4} \frac{l}{m} \Omega\left(b_{i / m} b_{j / m}+c_{i / m} c_{j / m}\right)$

$\left[\operatorname{sgn}(l-i+j) \delta_{|l-i+j|}^{k}\right.$

$\left.+\operatorname{sgn}(l+i-j) \delta_{|l+i-j|}^{k}\right] \delta_{l}^{r}$

$g_{13}^{(2)}(i, j, l, k, r)=\frac{2}{N^{2}} \dot{a}_{0}^{(m)} a_{0}^{(m)} \delta_{i}^{k} \delta_{i+N}^{r}+\frac{1}{2 N} \dot{a}_{0}^{(m)} b_{j / m}$

$\left[\operatorname{sgn}(i-j) \delta_{|i-j|}^{k}+\delta_{i+j}^{k}\right] \delta_{i+N}^{q}$

$g_{14}^{(2)}(i, j, l, k, r)=\frac{1}{2 N} \dot{a}_{0}^{(m)} b_{i / m}$

$\left[\delta_{i+j}^{k}-\operatorname{sgn}(i-j) \delta_{|i-j|}^{k}\right] \delta_{j+N}^{r}$

$g_{15}^{(2)}(i, j, l, k, r)=\frac{1}{N} a_{0}^{(m)}\left(\dot{b}_{l / m}+\frac{l}{m} \Omega c_{l / m}\right)$
Fig. 1 Frequency-amplitude curves of harmonic term based on 100 harmonic terms for period-1, period-3 and period-5 motions in the van der Pol oscillator $\left(\alpha_{1}=5.0, \alpha_{2}=5.0, \alpha_{3}=1.0, Q_{0}=5.0\right)$

$$
\left[\delta_{l+i}^{k}-\operatorname{sgn}(l-i) \delta_{|l-i|}^{k}\right] \delta_{i+N}^{r}
$$

$g_{16}^{(2)}(i, j, l, k, r)=\frac{1}{N} \frac{l}{m} \Omega a_{0}^{(m)} c_{i / m}$

$$
\left[\delta_{l+i}^{k}-\operatorname{sgn}(l-i) \delta_{|l-i|}^{k}\right] \delta_{l+N}^{r}
$$

$g_{17}^{(2)}(i, j, l, k, r)=\frac{1}{2} b_{j / m}\left(\dot{b}_{l / m}+\frac{l}{m} \Omega c_{l / m}\right)$

$$
\left[\delta_{l+i+j}^{k}-\operatorname{sgn}(l-i-j) \delta_{|l-i-j|}^{k}\right] \delta_{i+N}^{r}
$$

$g_{18}^{(2)}(i, j, l, k, r)=\frac{1}{4} \frac{l}{m} \Omega\left(b_{i / m} c_{j / m}+b_{j / m} c_{i / m}\right)$

$\left[\delta_{l+i+j}^{k}-\operatorname{sgn}(l-i-j) \delta_{|l-i-j|}^{k}\right] \delta_{l+N}^{r}$

$g_{19}^{(2)}(i, j, l, k, r)=-\frac{1}{2} c_{j / m}\left(\dot{c}_{l / m}-\frac{l}{m} \Omega b_{l / m}\right)$

$\left[\delta_{l+i+j}^{k}+\operatorname{sgn}(l-i-j) \delta_{|l-i-j|}^{k}\right] \delta_{i+N}^{r}$

$g_{20}^{(2)}(i, j, l, k, r)=\frac{1}{2} c_{j / m}\left(\dot{c}_{l / m}-\frac{l}{m} \Omega b_{l / m}\right)$

$\left[\operatorname{sgn}(l-i+j) \delta_{|l-i+j|}^{k}\right.$

$\left.+\operatorname{sgn}(l+i-j) \delta_{|l+i-j|}^{k}\right] \delta_{i+N}^{r}$

$g_{21}^{(2)}(i, j, l, k, r)=\frac{1}{4} \frac{l}{m} \Omega\left(b_{i / m} c_{j / m}-b_{j / m} c_{i / m}\right)$

$\left[\operatorname{sgn}(l-i+j) \delta_{|l-i+j|}^{k}\right.$

$\left.-\operatorname{sgn}(l+i-j) \delta_{|l+i-j|}^{k}\right] \delta_{l+N}^{r}$

for $r=0,1, \ldots, 2 N$.

$\mathbf{H}=\frac{\partial \mathbf{g}^{(m)}}{\partial \mathbf{z}_{1}^{(m)}}=\left(\mathbf{H}^{(0)}, \mathbf{H}^{(c)}, \mathbf{H}^{(s)}\right)^{\mathrm{T}}$ 


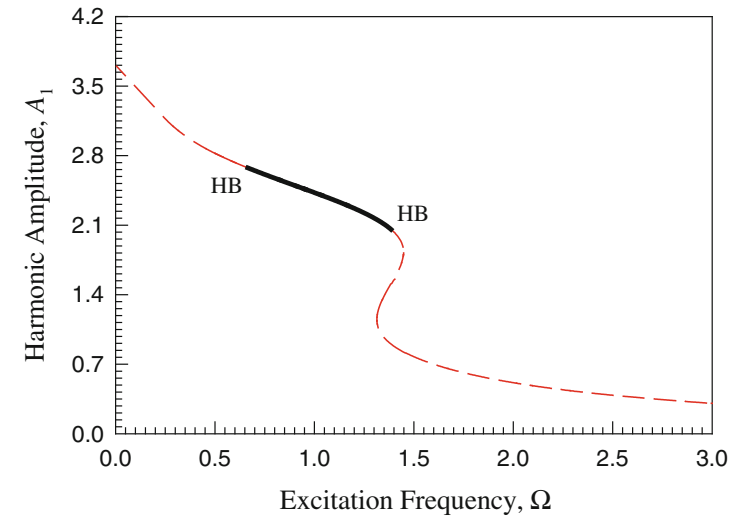

(i)

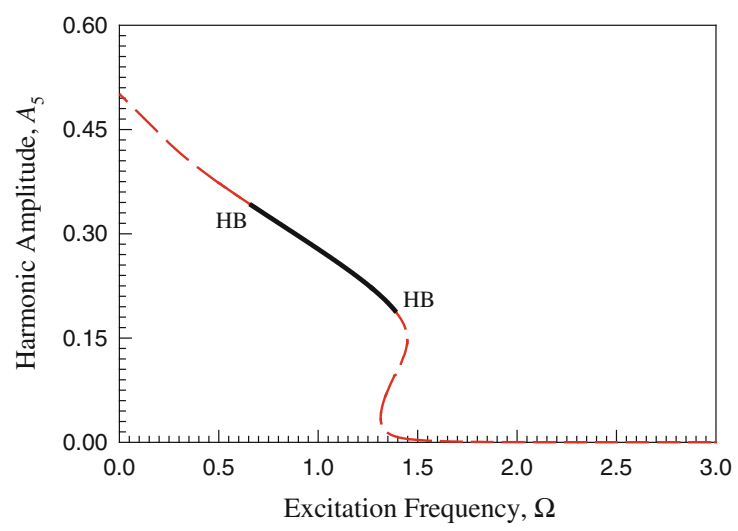

(iii)

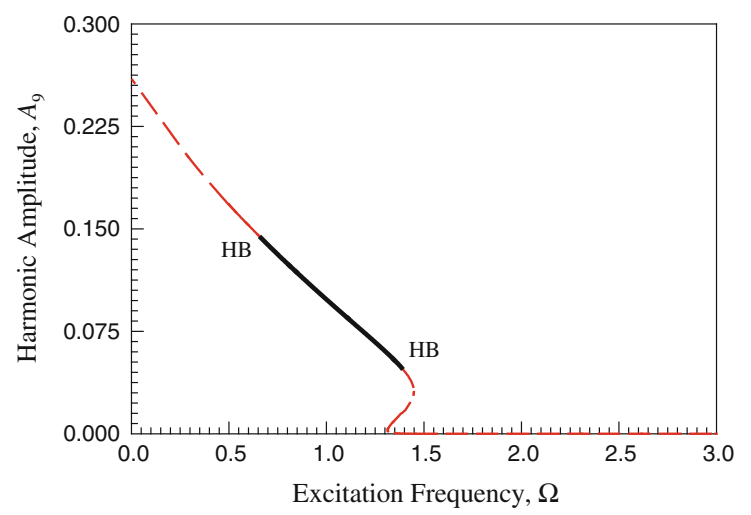

(v)

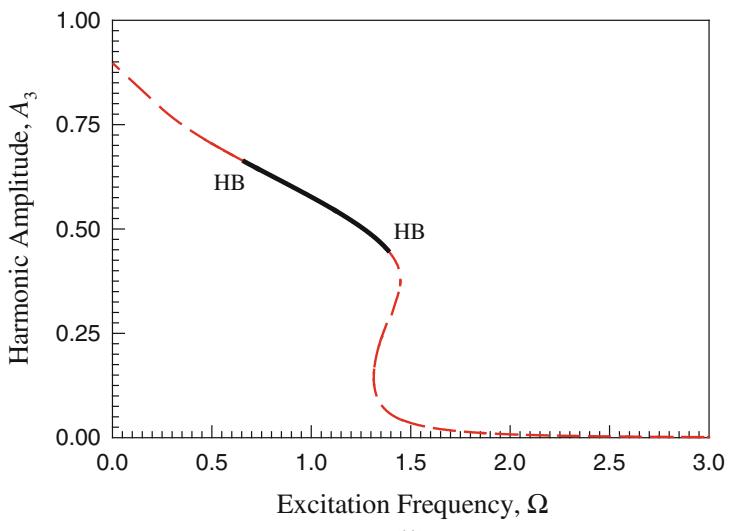

(ii)

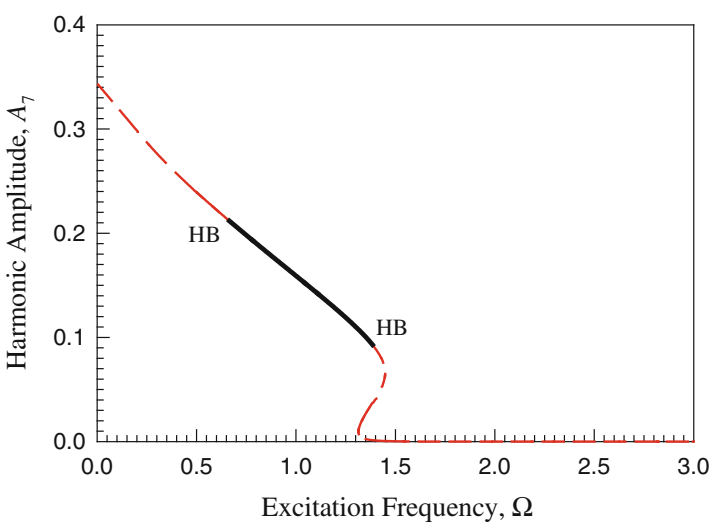

(iv)

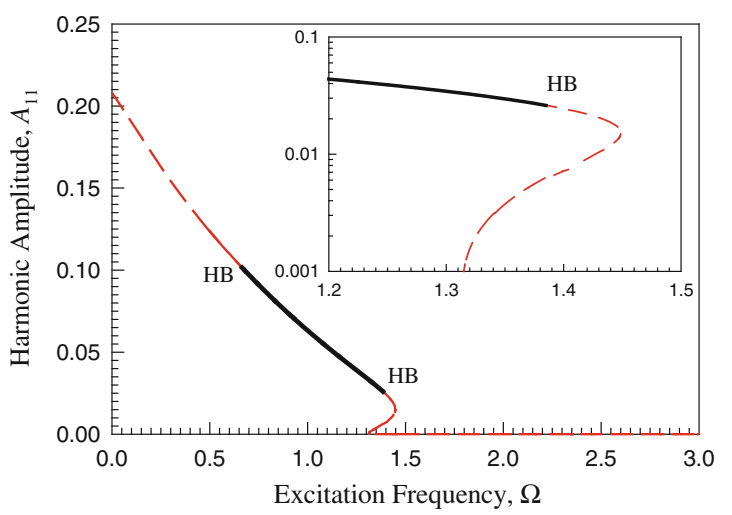

(vi)

Fig. 2 Frequency-amplitude curves of harmonic terms based on 90 harmonic terms for period-1 motion in the van der Pol oscillator: (i)-(x) $A_{2 m-1}(m=1,2, \ldots, 10)$, (xi) $A_{55}$ and (xii) $A_{89}\left(\alpha_{1}=5.0, \alpha_{2}=5.0, \alpha_{3}=1.0, Q_{0}=5.0\right)$

where

$$
\begin{aligned}
\mathbf{H}^{(0)} & =\left(H_{0}^{(0)}, H_{1}^{(0)}, \ldots, H_{2 N}^{(0)}\right), \\
\mathbf{H}^{(c)} & =\left(\mathbf{H}_{1}^{(c)}, \mathbf{H}_{2}^{(c)}, \ldots, \mathbf{H}_{N}^{(c)}\right)^{\mathrm{T}}, \\
\mathbf{H}^{(s)} & =\left(\mathbf{H}_{1}^{(s)}, \mathbf{H}_{2}^{(s)}, \ldots, \mathbf{H}_{N}^{(s)}\right)^{\mathrm{T}}
\end{aligned}
$$

for $N=1,2, \ldots \infty$, with

$$
\begin{aligned}
\mathbf{H}_{k}^{(c)} & =\left(H_{k 0}^{(c)}, H_{k 1}^{(c)}, \ldots, H_{k(2 N)}^{(c)}\right), \\
\mathbf{H}_{k}^{(s)} & =\left(H_{k 0}^{(s)}, H_{k 1}^{(s)}, \ldots, H_{k(2 N)}^{(s)}\right)
\end{aligned}
$$

for $k=1,2, \ldots N$. The corresponding components are

$$
\begin{aligned}
& H_{r}^{(0)}=\alpha_{1} \delta_{0}^{r}-\alpha_{2} h_{r}^{(0)} \\
& H_{k r}^{(c)}=-2\left(\frac{k \Omega}{m}\right) \delta_{k+N}^{r}+\alpha_{1} \delta_{k}^{r}-\alpha_{2} h_{k r}^{(c)},
\end{aligned}
$$




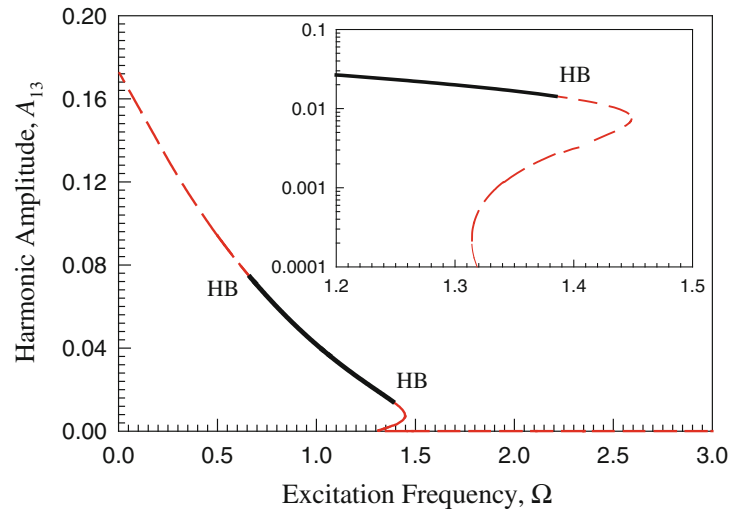

(vii)

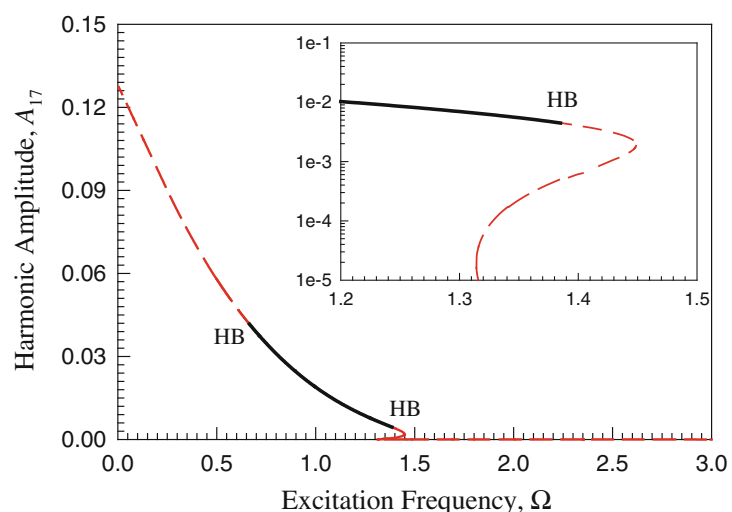

(ix)

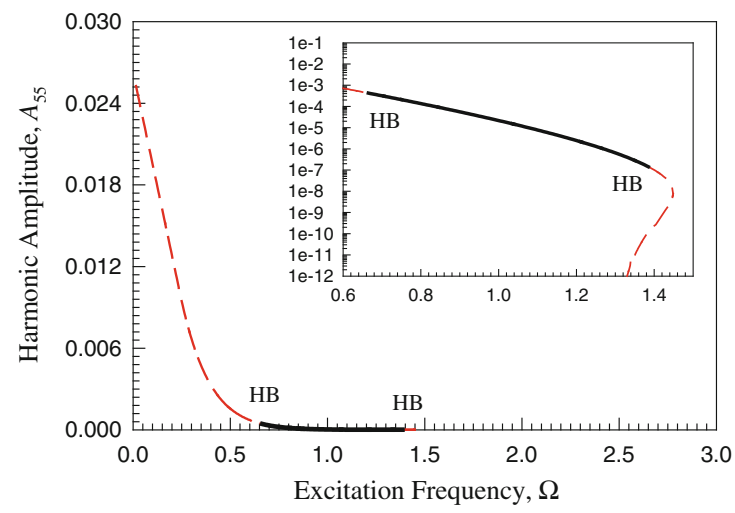

(xi)

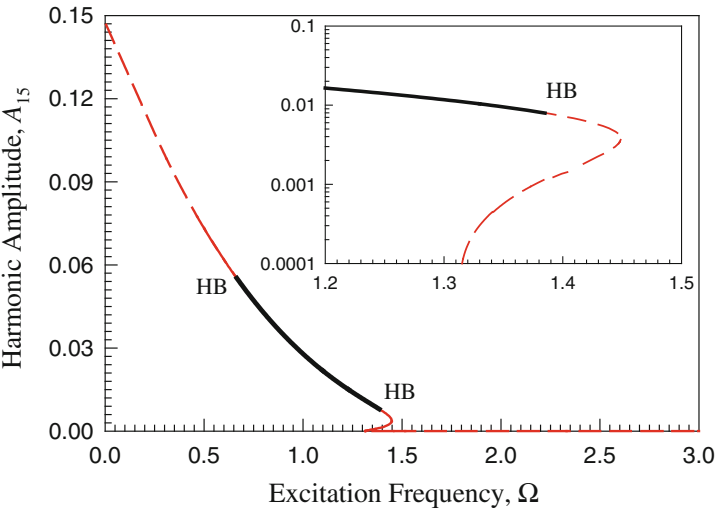

(viii)

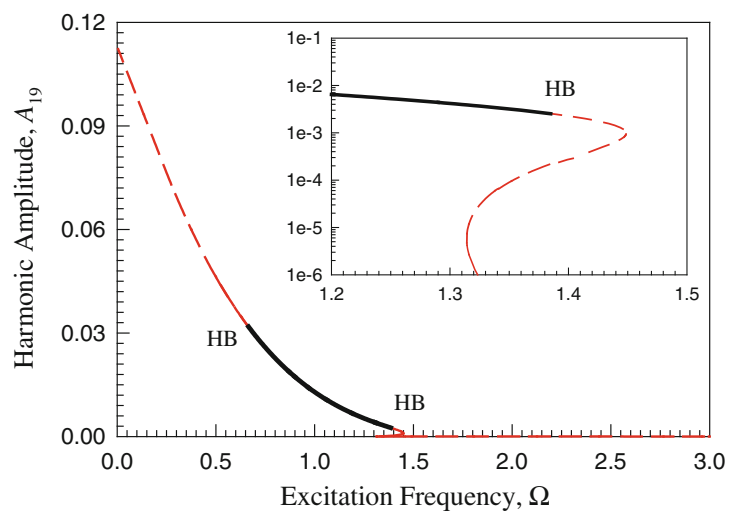

(x)

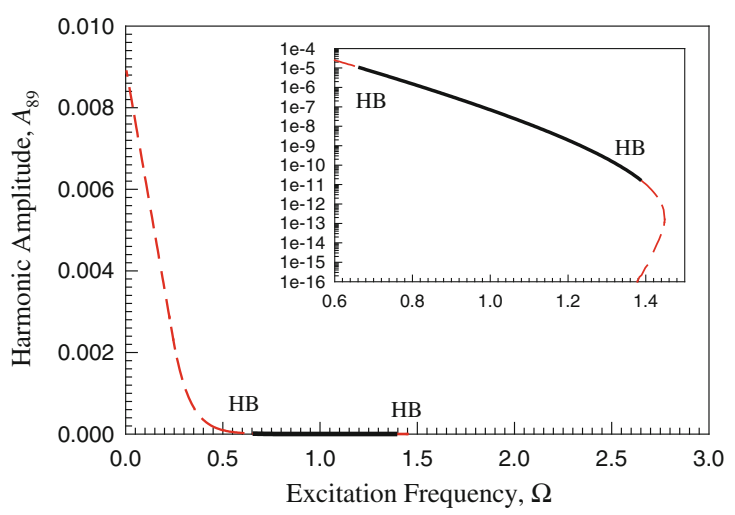

(xii)

Fig. 2 continued

$H_{k r}^{(s)}=2\left(\frac{k \Omega}{m}\right) \delta_{k}^{r}+\alpha_{1} \delta_{k+N}^{r}-\alpha_{2} h_{k r}^{(s)}$

for $r=0,1, \ldots, 2 N$.

$h_{r}^{(0)}=\left(a_{0}^{(m)}\right)^{2} \delta_{0}^{r}+\sum_{n=1}^{6} \sum_{l=1}^{N} \sum_{j=1}^{N} \sum_{i=1}^{N} h_{n}^{(0)}(i, j, l, r)$

with

$h_{1}^{(0)}(i, j, l, r)=\frac{1}{2 N} b_{i / m} b_{j / m} \delta_{i-j}^{0} \delta_{0}^{r}$

$$
\begin{aligned}
h_{2}^{(0)}(i, j, l, r)= & \frac{1}{2 N} c_{i / m} c_{j / m} \delta_{i-j}^{0} \delta_{0}^{r}+\frac{1}{N} a_{0}^{(m)} b_{i / m} \delta_{l-i}^{0} \delta_{l}^{r} \\
h_{3}^{(0)}(i, j, l, r)= & \frac{1}{4}\left(b_{i / m} b_{j / m}-c_{i / m} c_{j / m}\right) \delta_{l-i-j}^{0} \delta_{l}^{r} \\
h_{4}^{(0)}(i, j, l, r)= & \frac{1}{4}\left(b_{i / m} b_{j / m}+c_{i / m} c_{j / m}\right) \\
& \left(\delta_{l-i+j}^{0}+\delta_{l+i-j}^{0}\right) \delta_{l}^{r} \\
h_{5}^{(0)}(i, j, l, r)= & \frac{1}{N} a_{0}^{(m)} c_{i / m} \delta_{l-i}^{0} \delta_{l+N}^{r} \\
& +\frac{1}{4}\left(b_{j / m} c_{i / m}+b_{i / m} c_{j / m}\right) \delta_{l-i-j}^{0} \delta_{l+N}^{r}
\end{aligned}
$$




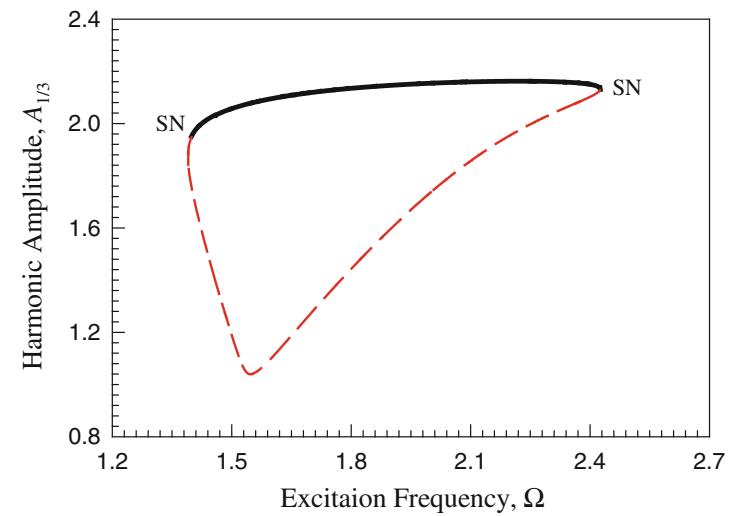

(i)

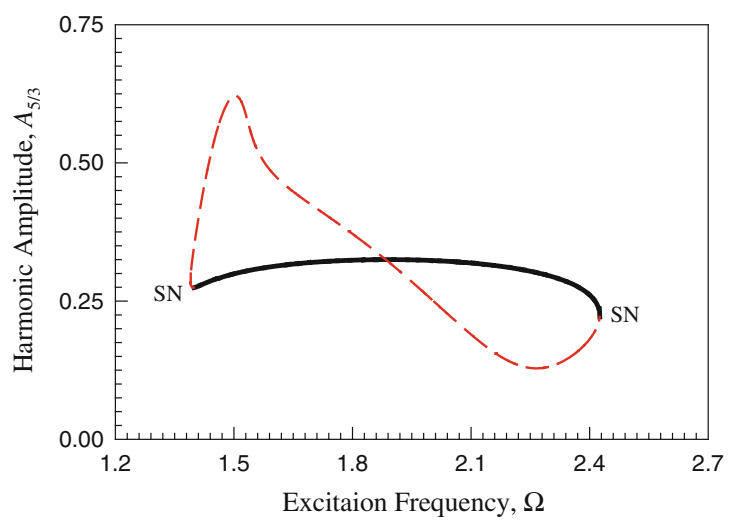

(iii)

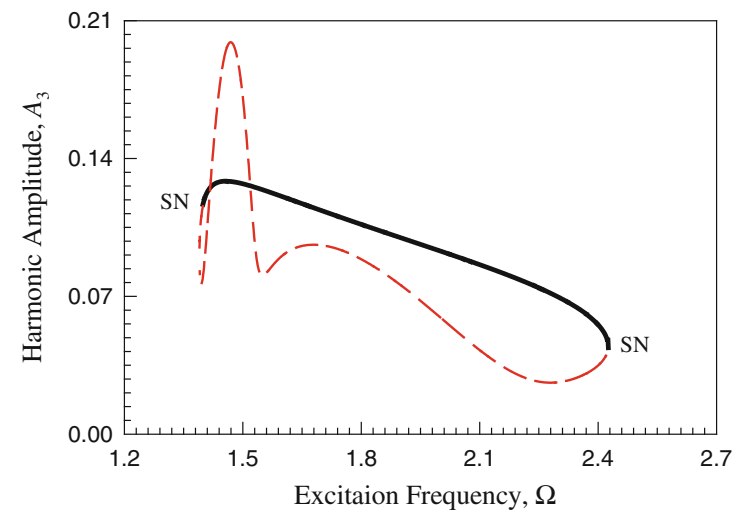

(v)

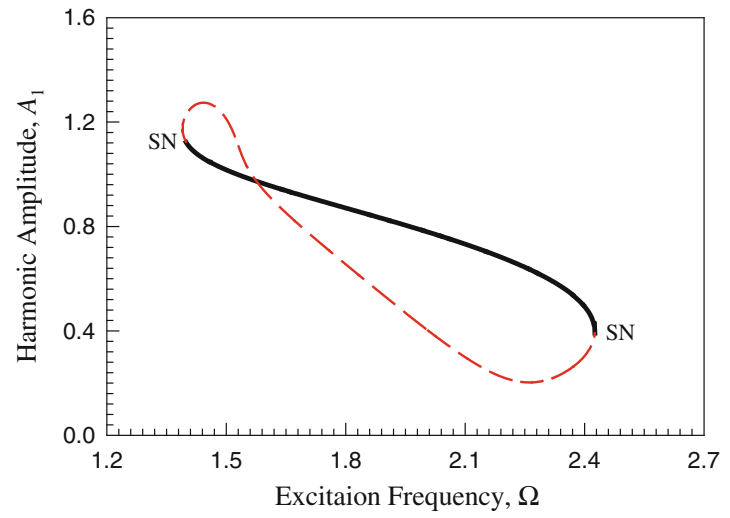

(ii)

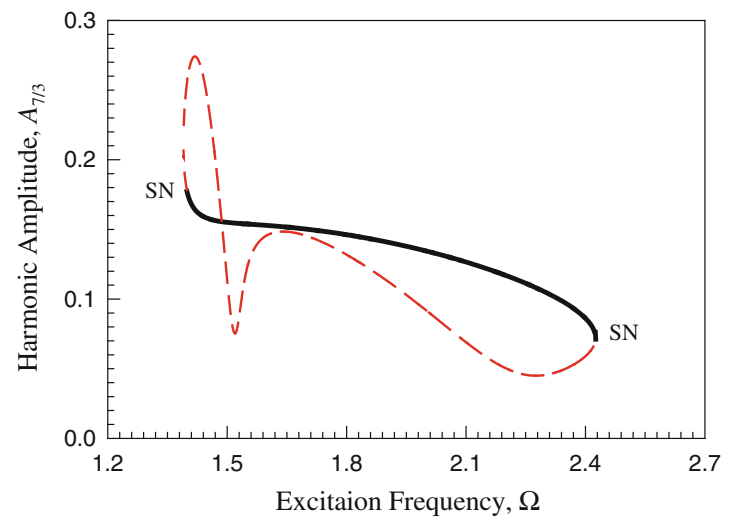

(iv)

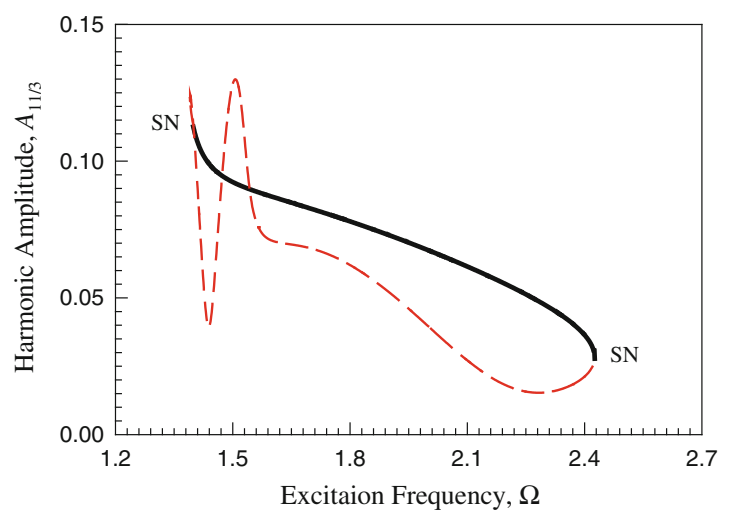

(vi)

Fig. 3 Frequency-amplitude curves of harmonic terms based on 60 harmonic terms for period-3 motion in the van der Pol oscillator: (i)-(xi) $A_{(2 m-1) / 3}(m=1,2, \ldots, 11)$ and (xii) $A_{59 / 3}\left(\alpha_{1}=5.0, \alpha_{2}=5.0, \alpha_{3}=1.0, Q_{0}=5.0\right)$

$$
\begin{aligned}
h_{6}^{(0)}(i, j, l, r)= & \frac{1}{4}\left(b_{j / m} c_{i / m}-b_{i / m} c_{j / m}\right) \\
& \left(\delta_{l-i+j}^{0}+\delta_{l+i-j}^{0}\right) \delta_{l+N}^{r}
\end{aligned}
$$

and

$h_{k r}^{(c)}=\sum_{n=1}^{9} \sum_{l=1}^{N} \sum_{j=1}^{N} \sum_{i=1}^{N} h_{n}^{(1)}{ }_{(i, j, l, k, r)}$ with

$$
\begin{aligned}
& h_{1}^{(1)}(i, j, l, k, r)=2 \frac{1}{N^{2}} \dot{a}_{0}^{(m)} b_{i / m} \delta_{l}^{k} \delta_{0}^{r} \\
& h_{2}^{(1)}(i, j, l, k, r)=\frac{1}{2 N} b_{i / m} b_{j / m}\left(\delta_{|l-i|}^{k}+\delta_{l+i}^{k}\right) \delta_{0}^{r} \\
& h_{3}^{(1)}(i, j, l, k, r)=\frac{1}{2 N} c_{i / m} c_{j / m}\left(\delta_{|l-i|}^{k}-\delta_{l+i}^{k}\right) \delta_{0}^{r}
\end{aligned}
$$




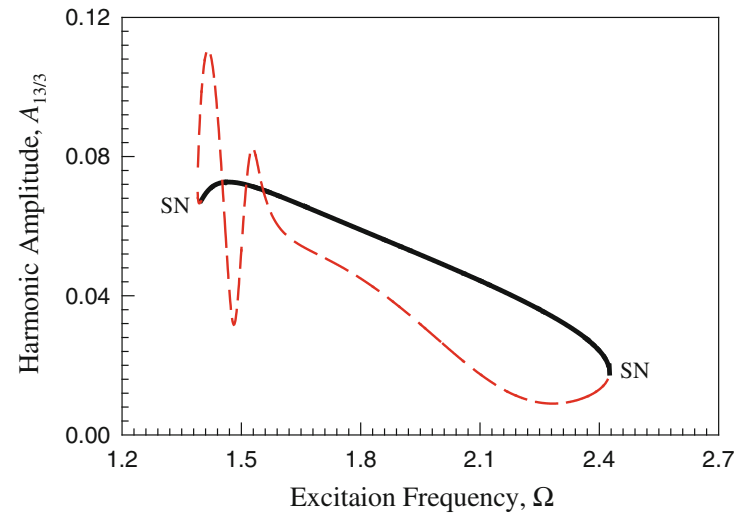

(vii)

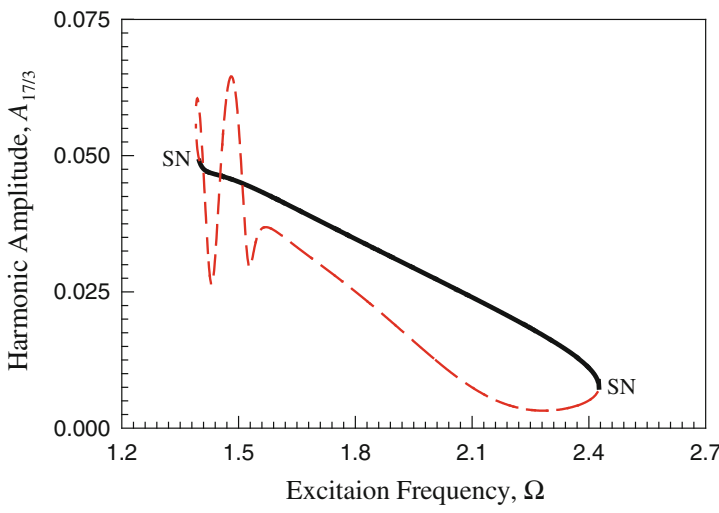

(ix)

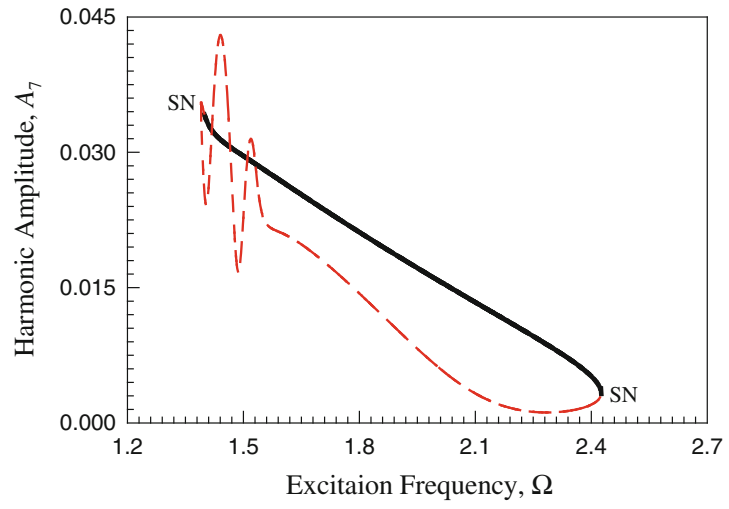

(xi)

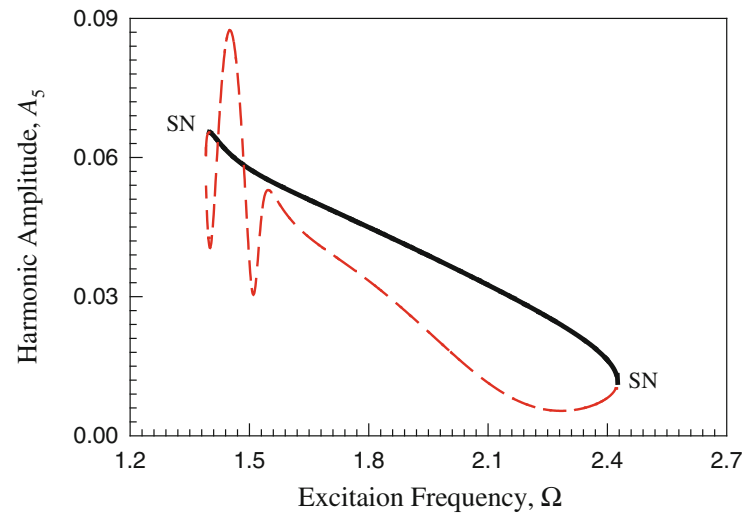

(viii)

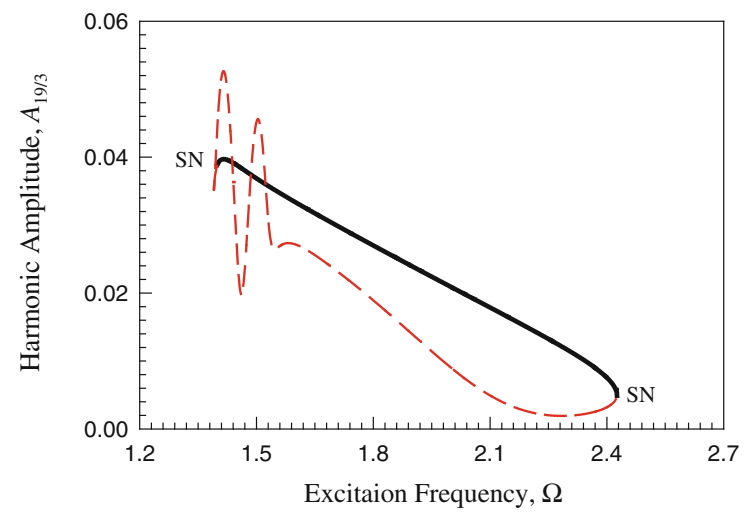

(x)

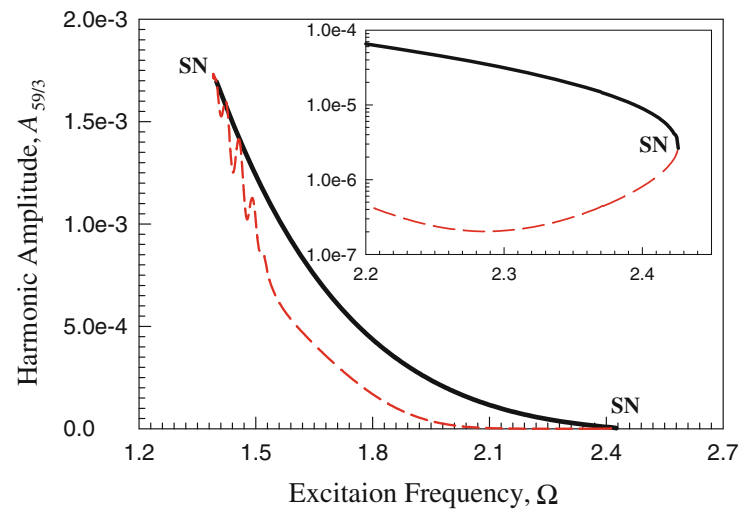

(xii)

Fig. 3 continued

$$
\begin{aligned}
h_{4}^{(1)}(i, j, l, k, r)= & \frac{1}{N^{2}}\left(a_{0}^{(m)}\right) \delta_{l}^{k} \delta_{l}^{r}+\frac{1}{N} a_{0}^{(m)} b_{i / m} \\
& \left(\delta_{|l-i|}^{k}+\delta_{l+i}^{k}\right) \delta_{l}^{r} \\
h_{5}^{(1)}(i, j, l, k, r)= & \frac{1}{4}\left(b_{i / m} b_{j / m}-c_{i / m} c_{j / m}\right) \\
& \left(\delta_{|l-i-j|}^{k}+\delta_{l+i+j}^{k}\right) \delta_{l}^{r} \\
h_{6}^{(1)}(i, j, l, k, r)= & \frac{1}{4}\left(b_{i / m} b_{j / m}+c_{i / m} c_{j / m}\right) \\
& \left(\delta_{|l-i+j|}^{k}+\delta_{|l+i-j|}^{k}\right) \delta_{l}^{r}
\end{aligned}
$$

$h_{7}^{(1)}(i, j, l, k, r)=\frac{1}{N} a_{0}^{(m)} c_{i / m}\left(\delta_{|l-i|}^{k}-\delta_{l+i}^{k}\right) \delta_{l+N}^{r}$ $h_{8}^{(1)}(i, j, l, k, r)=\frac{1}{4}\left(b_{j / m} c_{i / m}+b_{i / m} c_{j / m}\right)$$$
\left(\delta_{|l-i-j|}^{k}-\delta_{l+i+j}^{k}\right) \delta_{l+N}^{r}
$$$$
h_{9}^{(1)}(i, j, l, k, r)=\frac{1}{4}\left(b_{j / m} c_{i / m}-b_{i / m} c_{j / m}\right)
$$$$
\left(\delta_{|l-i+j|}^{k}-\delta_{|l+i-j|}^{k}\right) \delta_{l+N}^{r}
$$ 
and

$h_{k r}^{(s)}=\sum_{n=1}^{6} \sum_{l=1}^{N} \sum_{j=1}^{N} \sum_{i=1}^{N} h_{n}^{(2)}(i, j, l, k, r)$

with

$h_{1}^{(2)}(i, j, l, k, r)=\frac{2}{N^{2}} a_{0}^{(m)} c_{i / m} \delta_{i}^{k} \delta_{0}^{r}$

$h_{2}^{(2)}(i, j, l, k, r)=\frac{1}{2 N} b_{j / m} c_{i / m}\left[\operatorname{sgn}(i-j) \delta_{|i-j|}^{k}+\delta_{i+j}^{k}\right] \delta_{0}^{r}$

$h_{3}^{(2)}(i, j, l, k, r)=\frac{1}{2 N} b_{i / m} c_{j / m}\left[\delta_{i+j}^{k}-\operatorname{sgn}(i-j) \delta_{|i-j|}^{k}\right] \delta_{0}^{r}$

$h_{4}^{(2)}(i, j, l, k, r)=\frac{1}{N} a_{0}^{(m)} c_{i / m}\left[\delta_{l+i}^{k}-\operatorname{sgn}(l-i) \delta_{|l-i|}^{k}\right] \delta_{l}^{r}$

$h_{5}^{(2)}(i, j, l, k, r)=\frac{1}{4}\left(b_{i / m} c_{j / m}+b_{j / m} c_{i / m}\right)$

$\left[\delta_{l+i+j}^{k}-\operatorname{sgn}(l-i-j) \delta_{|l-i-j|}^{k}\right] \delta_{l}^{r}$

$h_{6}^{(2)}(i, j, l, k, r)=\frac{1}{4}\left(b_{i / m} c_{j / m}-b_{j / m} c_{i / m}\right)$

$\left[\operatorname{sgn}(l-i+j) \delta_{|l-i+j|}^{k}\right.$

$\left.-\operatorname{sgn}(l+i-j) \delta_{|l+i-j|}^{k}\right] \delta_{l}^{r}$

From Luo [18], the eigenvalues of $D \mathbf{f}^{(m)}\left(\mathbf{y}^{*(m)}\right)$ are classified as

$\left(n_{1}, n_{2}, n_{3} \mid n_{4}, n_{5}, n_{6}\right)$

The corresponding boundary between the stable and unstable solution is given by the saddle-node bifurcation and Hopf bifurcation.

\section{Frequency-amplitude characteristics}

The curves of harmonic amplitude varying with excitation frequency $\Omega$ are illustrated. The corresponding solution in Eq. (3) can be re-written as

$x^{*}(t)=a_{0}^{(m)}+\sum_{k=1}^{N} A_{k / m} \cos \left(\frac{k}{m} \Omega t-\varphi_{k / m}\right)$.

where the harmonic amplitude and phase are defined by

$A_{k / m} \equiv \sqrt{b_{k / m}^{2}+c_{k / m}^{2}}, \varphi_{k / m}=\arctan \frac{c_{k / m}}{b_{k / m}}$

The system parameters are

$\alpha_{1}=5.0, \alpha_{2}=5.0, \alpha_{3}=1.0, Q_{0}=5.0$

The frequency-amplitude curves for period-1, period-3 and period-5 motions based on 100 harmonic terms accordingly are presented in Fig. 1. The acronyms "HB" and "SN" are
Table 1 Input data for numerical illustrations $\left(\alpha_{1}=5.0, \alpha_{2}=\right.$ $5.0, \alpha_{3}=1.0, Q_{0}=5.0$ )

\begin{tabular}{llll}
\hline & $\Omega$ & Initial condition $\left(x_{0}, \dot{x}_{0}\right)$ & \\
\hline Fig. 5a-d & 1.385 & $(-1.781350,0.579786)$ & HB90 (P-1) \\
Fig. 6a-d & 2.3 & $(1.707910,0.330133)$ & HB60 (P-3) \\
Fig. 7a-d & 3.2 & $(-0.245784,0.098412)$ & HB100 (P-5) \\
\hline
\end{tabular}

used to represent the Hopf bifurcation and saddle-node bifurcation, respectively. Solid and dashed curves represent stable and unstable period-m motions. For the period-1 motion, the Hopf bifurcation is observed. After the Hopf bifurcation, the quasi-periodic motions exists and further the chaotic motions can be developed. For period-3 and period-5 motions, the saddle-node bifurcations are observed. After the saddle-node bifurcation, the period-3 and period-5 motions disappear. Such periodic motions are embedded in chaotic motions or switch to the other periodic motions. In Fig. 1, it is obviously observed that three periodic motions possess gaps. In such gaps, the quasi-periodic and chaotic motions will exist. For 100 harmonic terms, curves of harmonic frequencyamplitudes can be similarly presented. The curves shapes may be different, but with increasing harmonics order, the harmonic amplitudes will decrease.

For a better understanding of period-m motions, the comprehensive discussion of frequency-amplitude responses should be completed herein. In Fig. 2, the harmonic amplitudes $A_{2 m-1}(m=1,2, \ldots, 10), A_{55}$ and $A_{89}$ are presented. For $\Omega \in(0, \infty), A_{1}<4.0, A_{3}<1.0, A_{5}<5 \times 10^{-1}, A_{7}<$ $4 \times 10^{-1}, A_{7}<4 \times 10^{-1}, A_{9}<3 \times 10^{-1}, A_{11}<2 \times 10^{-1}$. For harmonic amplitude $A_{11}$, the zoomed window shows the stable period- 1 motion have $A_{11} \in(0.02,0.1)$. Similarly, $A_{13}<1.6 \times 10^{-1}$ but the stable period-1 motion with $A_{13} \in(0.02,0.07) ; A_{15}<1.6 \times 10^{-1}$ but the stable period1 motion with $A_{15} \in(0.02,0.07) ; A_{17}<1.3 \times 10^{-1}$ but the stable period-1 motion with $A_{17} \in(0.003,0.04) ; A_{19}<$ $1.2 \times 10^{-1}$ but the stable period-1 motion with $A_{19} \in$ (0.002, 0.03). For harmonic amplitude $A_{55}<2.5 \times 10^{-2}$, the stable period- 1 motion has $A_{55} \in\left(10^{-6}, 10^{-3}\right)$. For harmonic amplitude $A_{89}<9 \times 10^{-3}$, the stable period-1 motion has $A_{89} \in\left(10^{-10}, 10^{-5}\right)$. The Hopf bifurcations of period-1 motion occur at $\Omega \approx 0.665$ and $\Omega \approx 1.385$. After the two Hopf bifurcations, the quasi-periodic motions of the periodically excited, van der Pol oscillator are observed. For small excitation frequency, more harmonic terms should be involved in the finite Fourier series solution of period-1 motion.

For period-3 motion, the harmonic amplitudes $A_{(2 m-1) / 3}$ ( $m=1,2, \ldots, 11)$ and $A_{59}$ are presented for $\Omega \in$ $(1.3904,2.371)$ in Fig. 3. The stable period-3 motion is on the upper portion of the closed loop in $\Omega \in(1.3990,2.371)$. The harmonic amplitudes for the stable and unstable period- 


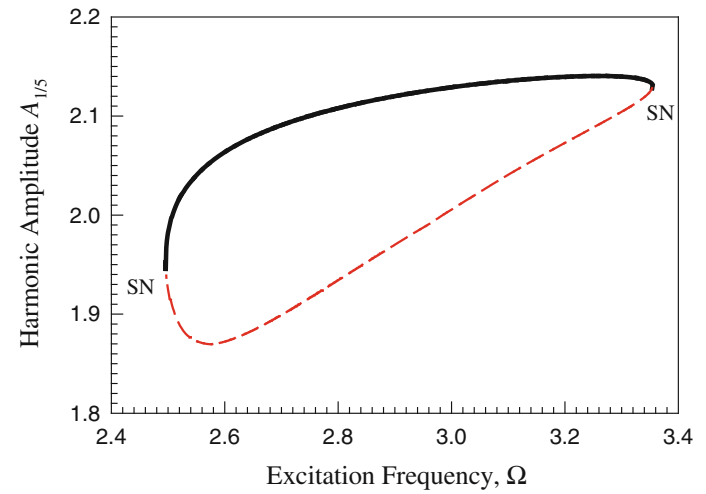

(i)

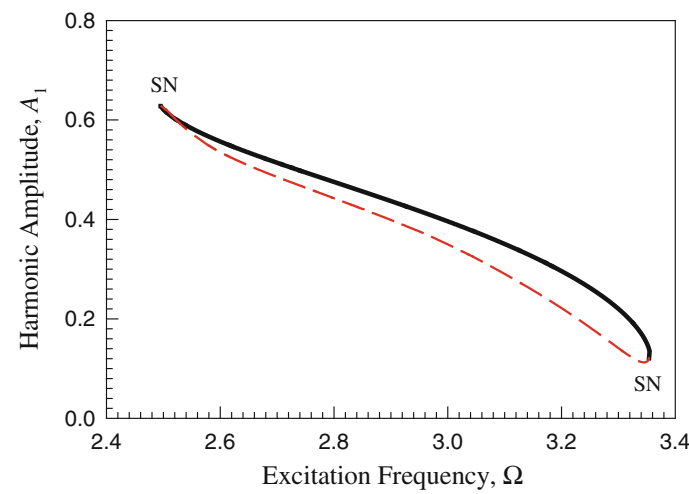

(iii)

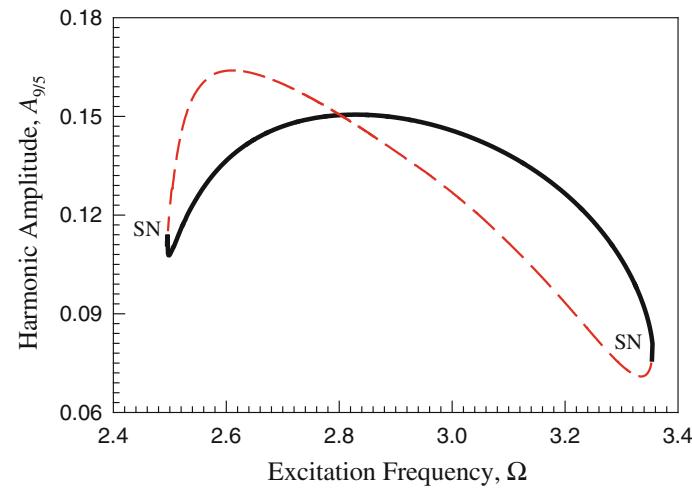

(v)

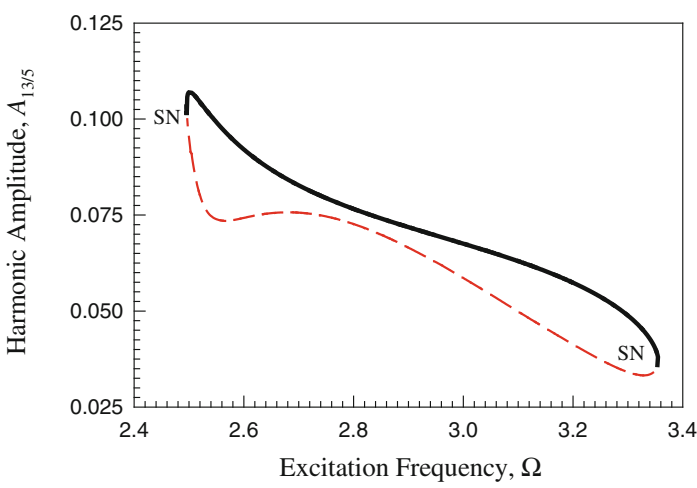

(vii)

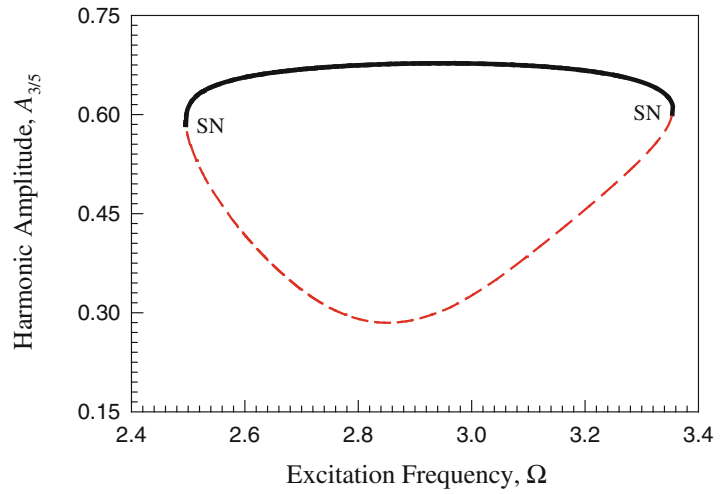

(ii)

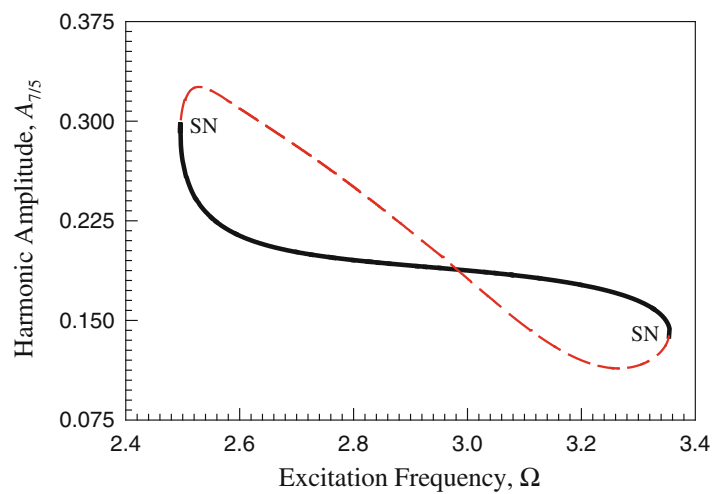

(iv)

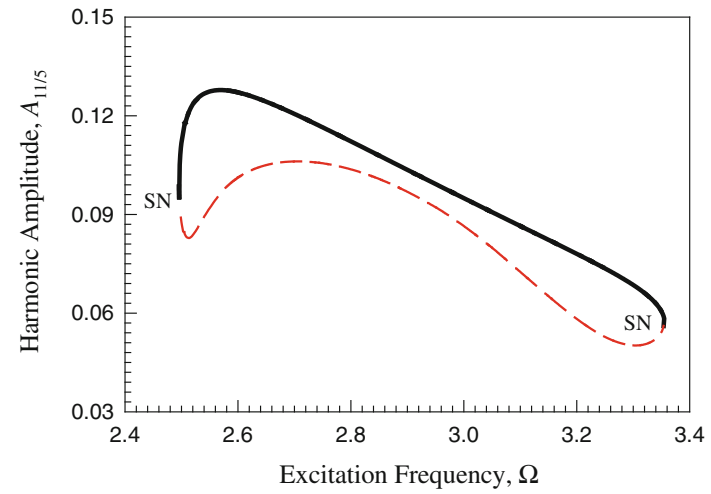

(vi)

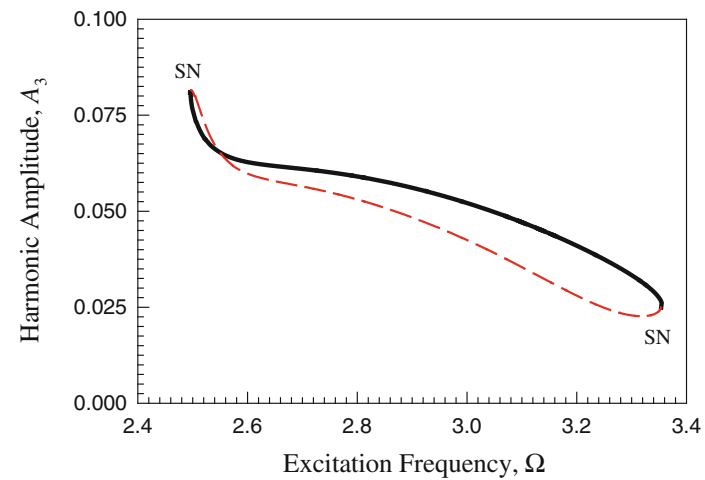

(viii)

Fig. 4 Frequency-amplitude curves of harmonic terms based on 100 harmonic terms for period-5 motion in the van der Pol oscillator: (i)-(xi) $A_{(2 m-1) / 5}(m=1,2, \ldots, 13)$ and (xii) $A_{99 / 5} \cdot\left(\alpha_{1}=5.0, \alpha_{2}=5.0, \alpha_{3}=1.0, Q_{0}=5.0\right)$ 


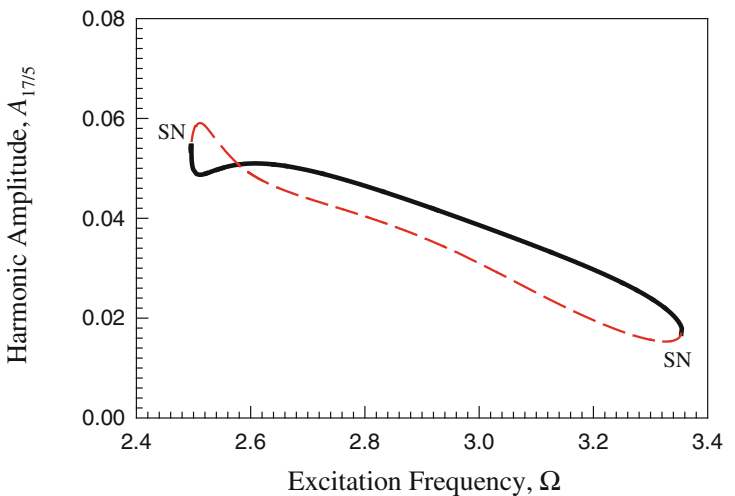

(ix)

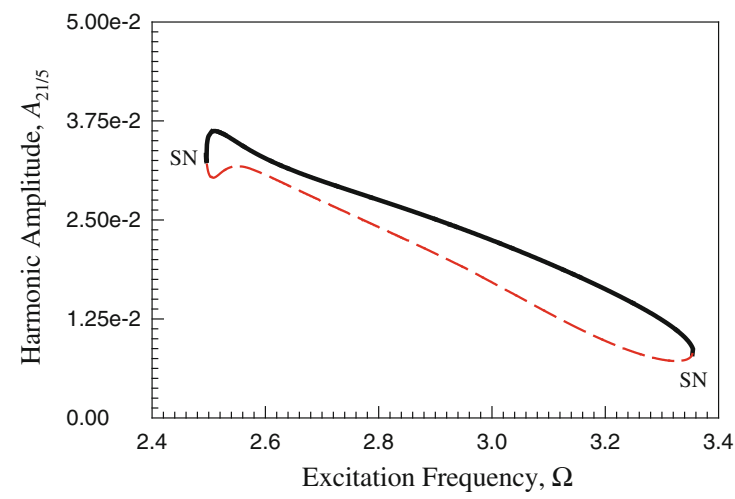

(xi)

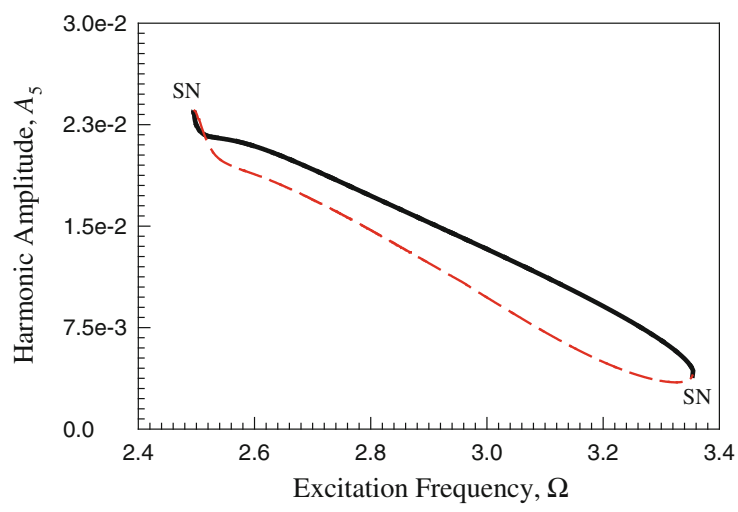

(xiii)

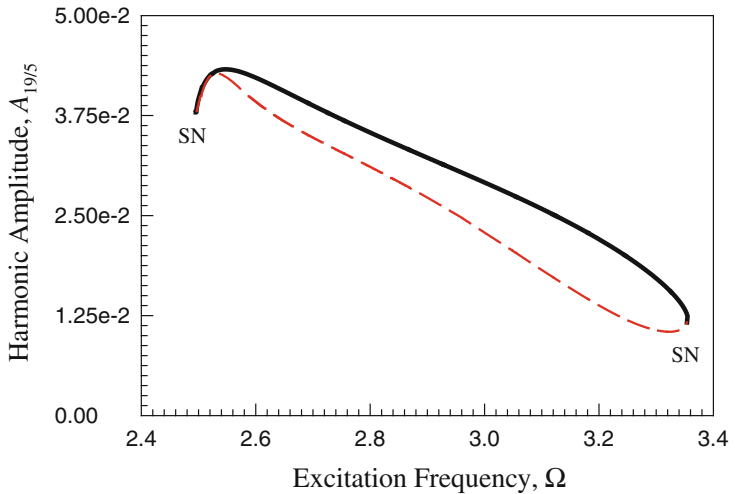

(x)

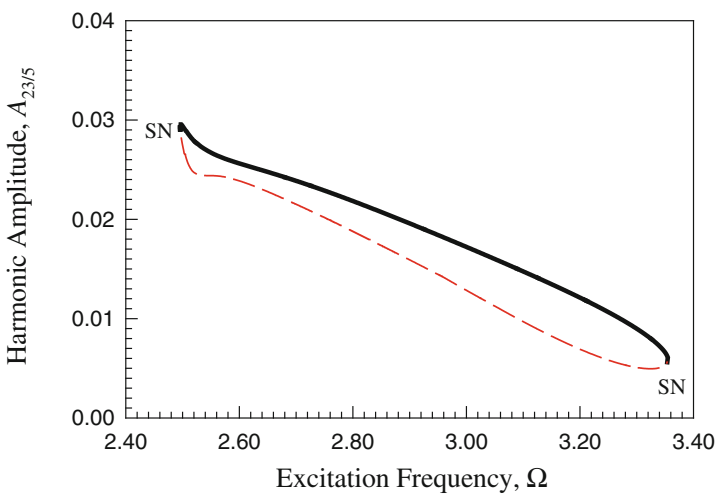

(xii)

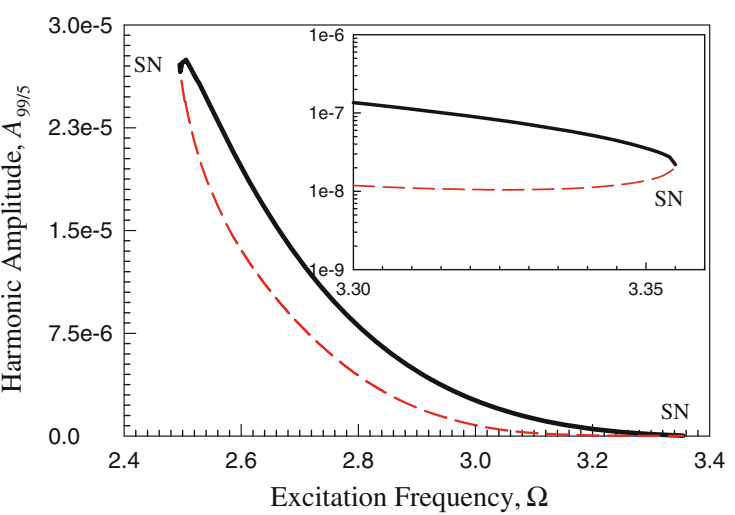

(xiv)

Fig. 4 continued

3 motion are $A_{1 / 3} \in(1.0,2.2), A_{1} \in(0.2,1.3), A_{5 / 3} \in$ $(0.15,0.7), A_{7 / 3} \in(0.05,0.3), A_{3} \in(0.03,0.21), A_{11 / 3} \in$ $(0.02,0.15), A_{13 / 3} \in(0.01,0.12), A_{15 / 3} \in(0.01,0.09)$, $A_{17 / 3} \in(0.003,0.07), A_{19 / 3} \in(0.002,0.06), A_{7} \in(0.001$, $0.05)$. To save page number, $A_{59 / 3} \in\left(10^{-7}, 2 \times 10^{-3}\right)$. For small excitation frequency, more harmonic terms should be involved in the finite Fourier series solution of period3 motion. The two saddle-node-bifurcations occur at $\Omega \approx$ 1.3990 and $\Omega \approx 2.371$. Once the period-3 motion disappears, the chaotic motions will be around. In other words, the period- 3 motion is embedded in chaotic motions of the periodically forced van der Pol oscillator.

For period-5 motion, the harmonic amplitudes $A_{(2 m-1) / 5}$ ( $m=1,2, \ldots, 13)$ and $A_{99 / 5}$ are presented for $\Omega \in$ $(2.4953,3.3540)$ in Fig. 4. The stable period-5 motion is on the upper portion of the closed loop in $\Omega \in(2.4953,3.3540)$. The harmonic amplitudes for the stable and unstable period5 motion are $A_{1 / 5} \in(1.8,2.2), A_{3 / 5} \in(0.3,0.7), A_{1} \in$ $(0.15,0.7), A_{7 / 5} \in(0.1,0.7), A_{9 / 5} \in(0.07,0.17), A_{11 / 5} \in$ (0.04, 0.13), $A_{13 / 5} \in(0.03,0.11), A_{3} \in(0.025,0.08)$, 
$A_{17 / 5} \in(0.02,0.06), A_{19 / 5} \in(0.01,0.045), A_{21 / 5} \in$ (0.007, 0.0375), $A_{23 / 5} \in\left(7 \times 10^{-3}, 3 \times 10^{-2}\right), A_{23 / 5} \in$ $\left(6 \times 10^{-3}, 2.5 \times 10^{-2}\right)$. To save page number, $A_{99 / 5} \in$ $\left(10^{-8}, 3 \times 10^{-5}\right)$. For small excitation frequency, more harmonic terms should be involved in the finite Fourier series solution of period-5 motion. The two saddle-nodebifurcations of period- 5 motions occur at $\Omega \approx 2.4953$ and $\Omega \approx 3.3540$. Once the period-5 motion disappears, the chaotic motions will also be around. In other words, the period-5 motion is embedded in chaotic motions of the periodically forced van der Pol oscillator.

\section{Numerical illustrations}

To illustrate period-m motions in the van der Pol oscillator, numerical simulations and analytical solutions will be presented. The initial conditions for numerical simulations are computed from approximate analytical solutions of periodic solutions. In all plots, circular symbols give approximate solutions, and solid curves give numerical simulation results.
The numerical solutions of periodic motions are generated via the symplectic scheme.

The trajectories and harmonic amplitudes of period-1, period- 3 and period- 5 motions are presented to verify approximate analytical periodic motions in such an oscillator. The displacement, velocity, trajectories and harmonic amplitude spectrums of period-1, period-3 and period-5 motions are presented for $\Omega=1.385,2.3,3.2$. The input data for numerical simulations is in Table 1. In Fig. 5, 90 harmonic terms (HB90) are used in the analytical solution of period-1 motion for $\Omega=1.385$. The displacement and velocity responses are presented in Fig. 5a, b, respectively. The analytical and numerical results of the period-1 motion match very well. The fast and slow movements of the van der Pol oscillator can be clearly observed. The main amplitudes are $A_{1} \approx 2.057854, A_{3} \approx 0.448938, A_{5} \approx 0.189691, A_{7} \approx$ $0.092938, A_{9} \approx 0.048419, A_{11} \approx 0.026053, A_{13} \approx$ 0.014295 . Since the excitation frequency is small, higher order harmonic terms should be in approximate solutions of period-1 motions. The trajectory for this period-1 motion has one cycle, and the higher order harmonic terms have significant contributions on the periodic motion.

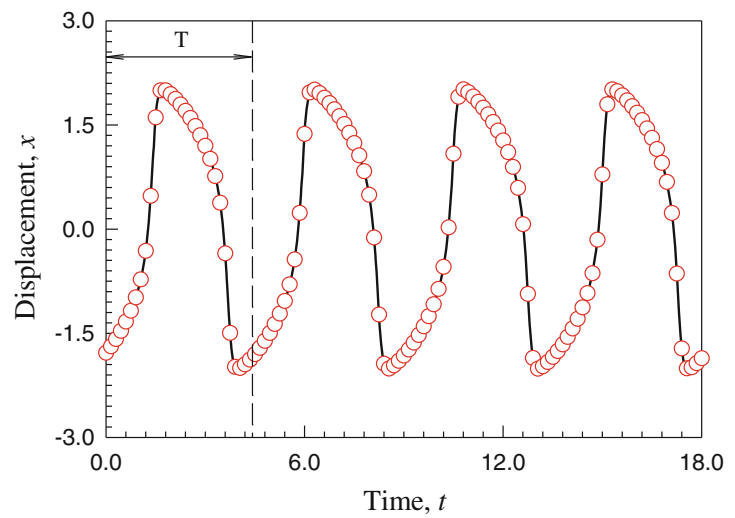

(a)

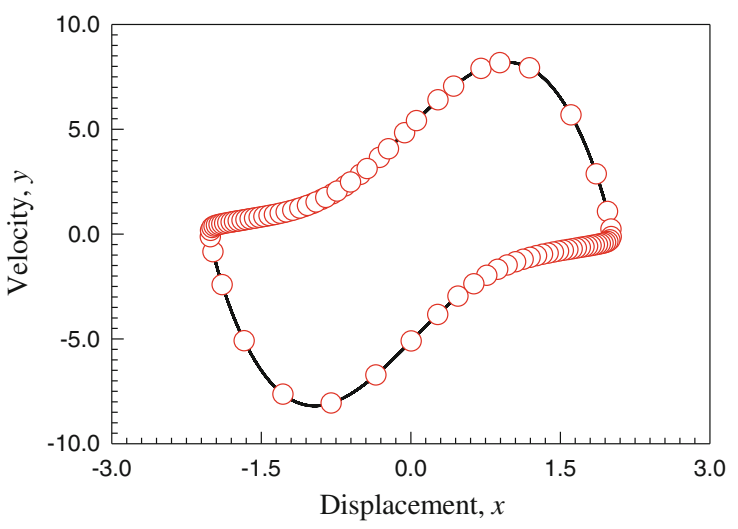

(c)

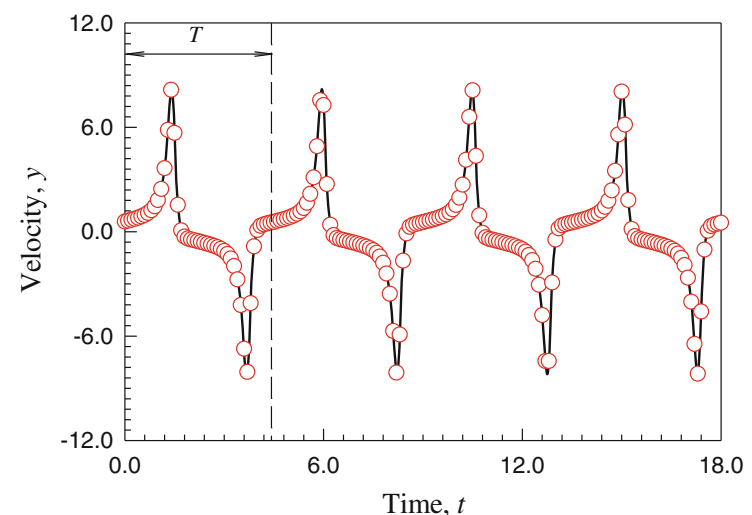

(b)

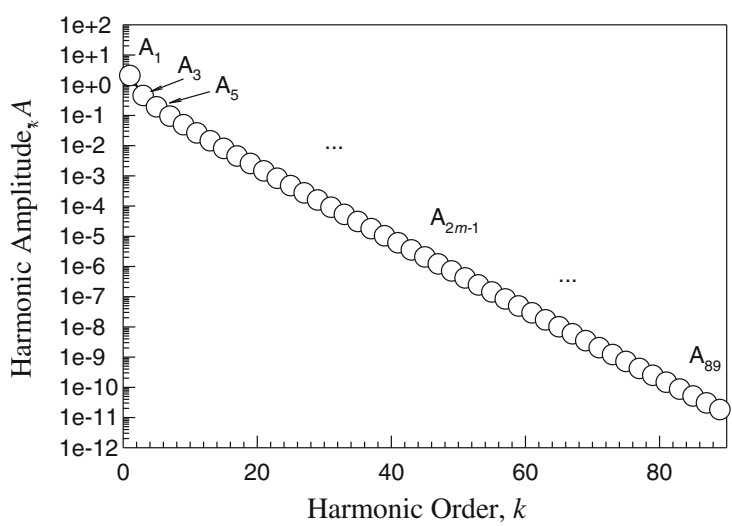

(d)

Fig. 5 Period-1 motion $(\Omega=1.138)$ : a displacement, b velocity, c trajectory and $\mathbf{d}$ amplitude. Initial condition $\left(x_{0}, \dot{x}_{0}\right)=(-1.781350$, 0.579786), $\left(\alpha_{1}=5.0, \alpha_{2}=5.0, \alpha_{3}=1.0, Q_{0}=5.0\right)$ 


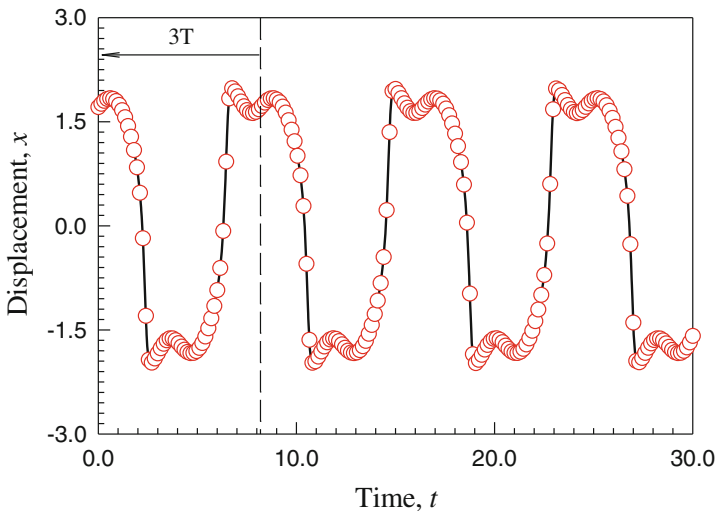

(a)

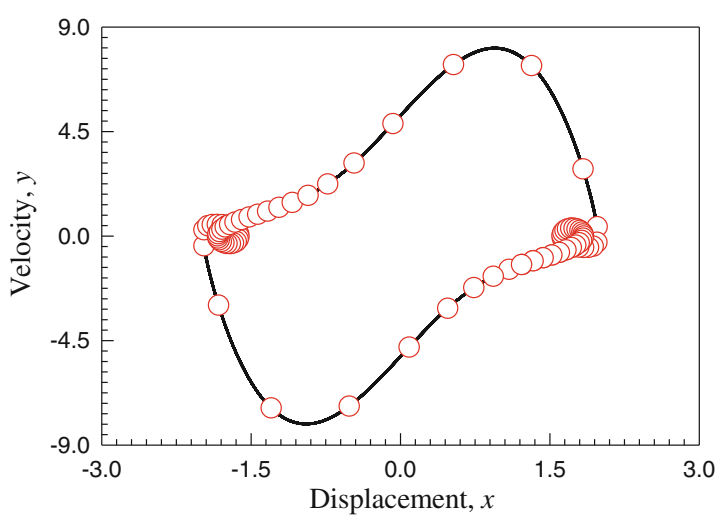

(c)

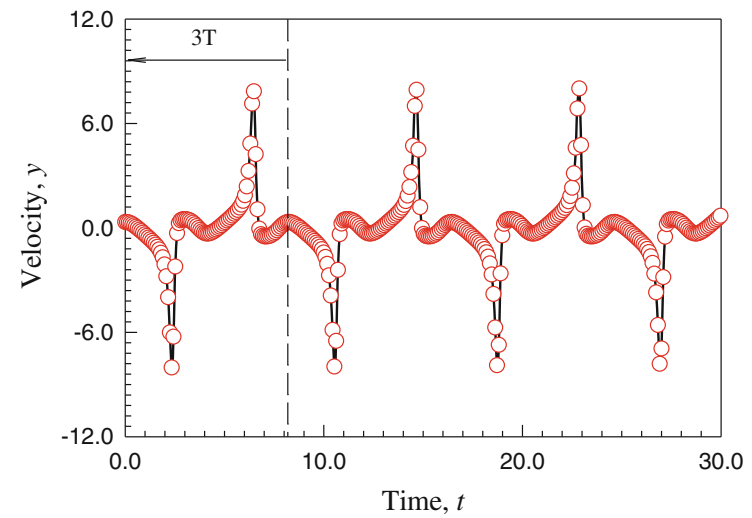

(b)

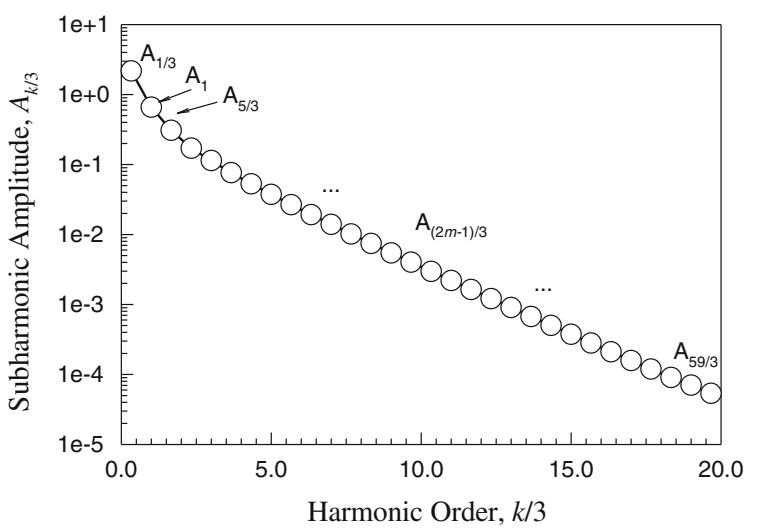

(d)

Fig. 6 Period-3 motion $(\Omega=2.3)$ : a displacement, b velocity, $\mathbf{c}$ trajectory and $\mathbf{d}$ amplitude. Initial condition $\left(x_{0}, \dot{x}_{0}\right)=(1.707910,0.330133)$, $\left(\alpha_{1}=5.0, \alpha_{2}=5.0, \alpha_{3}=1.0, Q_{0}=5.0\right)$

For the period-3 motion, 60 harmonic terms are used for approximate analytical solutions of period-3 motion, as shown in Fig. 6 for $\Omega=2.3$. The time-histories of displacement and velocity for period-3 motion are presented in Fig. 6a, b. Compared to the period-1 motion, the response curves for the slow moving portion are wavy. In Fig. 6c, the trajectory of period-3 motion for $\Omega=2.3$ is illustrated and the peiod- 3 motion possesses a large cycle with two small cycles, different from the period-1 motion. In Fig. 6d, the harmonic amplitudes are presented with their distribution $A_{1 / 3} \approx 2.162036, A_{1} \approx 0.657342, A_{5 / 3} \approx$ $0.306563, A_{7 / 3} \approx 0.170757, A_{3} \approx 0.113623, A_{11 / 3} \approx$ $0.076060, A_{13 / 3} \approx 0.052751, A_{5} \approx 0.037229, A_{17 / 3} \approx$ $0.026567, A_{19 / 3} \approx 0.019141, A_{7} \approx 0.013880, A_{23 / 3} \approx$ 0.010120 , “ $A_{(2 m-1) / 3} \sim 10^{-3}(m=13 \sim 19), 10^{-4}(m=$ $20 \sim 28)$, and $10^{-5}(m=29 \sim 30)$." The pattern of harmonic distributions is different from period-1 motion.

The period-5 motion is presented in Fig. 7 for $\Omega=3.2$ and the analytical solution is given by 100 harmonic terms (HB100). The analytical and numerical solutions for displacement and velocity responses of period-5 motion are shown in Fig. 7a, b, respectively. Compared to the period1 and period-3 motions, the response curves for the slow moving portion are more wavy. In Fig. 7c, the trajectory of a period-5 motion in phase plane is presented. The period5 motion has a big cycle with four small cycles at the two ends symmetrically. In Fig. 7d, the harmonic amplitudes are $A_{1 / 5} \approx 2.139456, A_{3 / 5} \approx 0.666350, A_{1} \approx$ $0.296105, A_{7 / 5} \approx 0.939406, A_{9 / 5} \approx 0.126531, A_{11 / 5} \approx$ $0.077993, A_{13 / 5} \approx 0.057344, A_{3} \approx 0.041025, A_{17 / 5} \approx$ $0.029733, A_{19 / 5} \approx 0.022050, A_{21 / 5} \approx 0.016265, A_{23 / 5} \approx$ $0.012120, " A_{(2 m-1) / 5} \sim 10^{-3}(m=13 \sim 20), 10^{-4}(m=$ $21 \sim 28), 10^{-5}(m=29 \sim 38), 10^{-6}(m=39 \sim$ 47), $10^{-7}(m=48 \sim 50) . "$

\section{Conclusions}

In this paper, the approximate analytical solutions of period$\mathrm{m}$ motions in the periodically forced van der Pol oscillator were obtained through the finite Fourier series expression. The corresponding stability and bifurcation analysis of such approximate solutions in such van der Pol oscillator were carried out, and the comprehensive discussion of frequencyamplitude characteristics were completed. Numerical illustrations for period-m motions in the periodically forced, van 


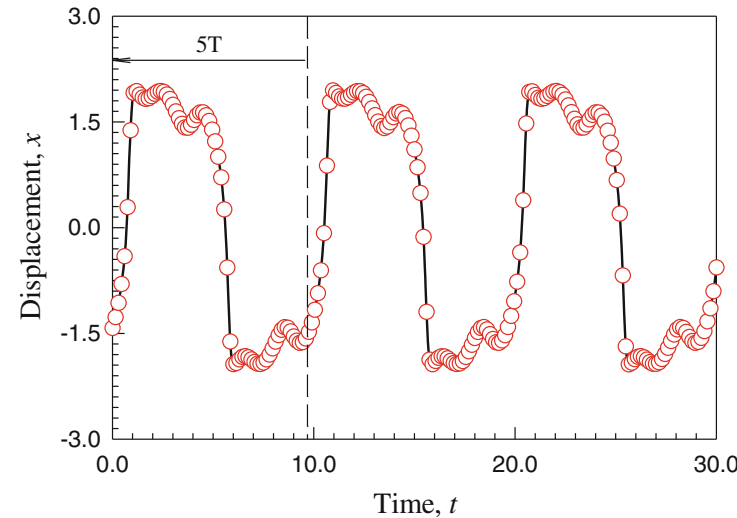

(a)

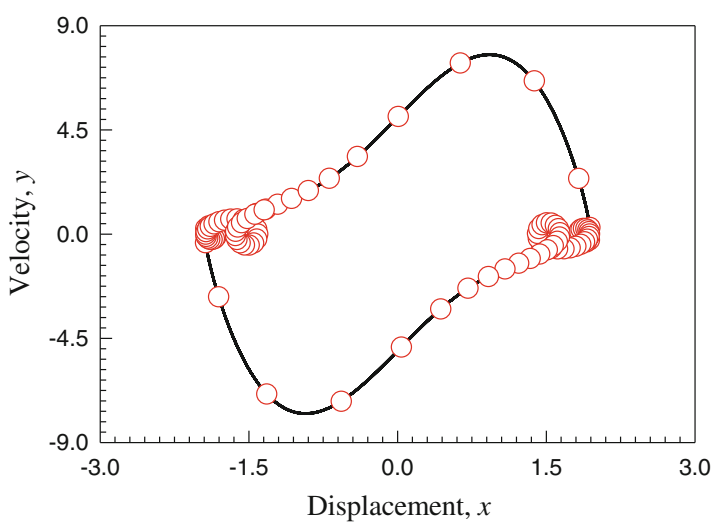

(c)

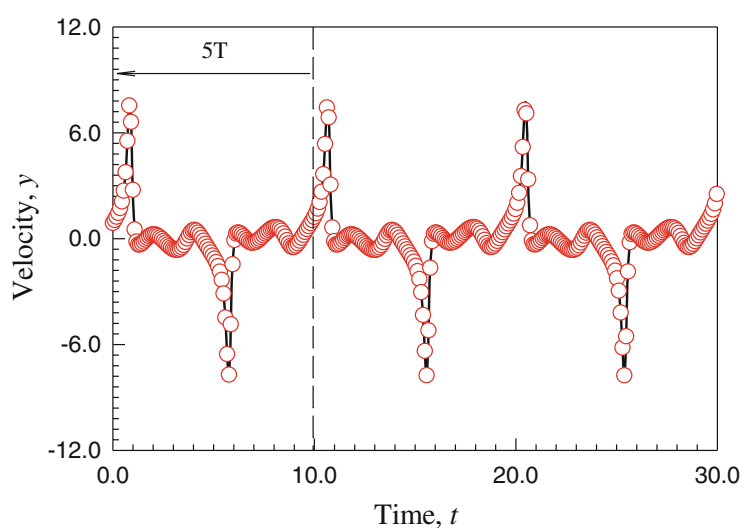

(b)

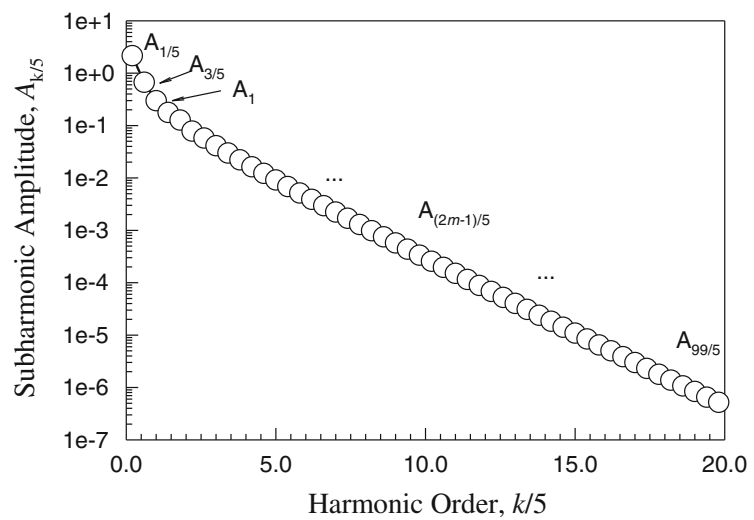

(d)

Fig. 7 Period-5 motion $(\Omega=3.2)$ : a displacement, b velocity, c trajectory and d amplitude. Initial condition $\left(x_{0}, \dot{x}_{0}\right)=(-0.245784$, $0.098412),\left(\alpha_{1}=5.0, \alpha_{2}=5.0, \alpha_{3}=1.0, Q_{0}=5.0\right)$

der Pol oscillator were carried out to verify the approximate solutions. The period-m motions are individually embedded in the quasi-periodic and chaotic motions.

\section{References}

1. Luo ACJ, Lakeh AB (2013) Period-m motions in a periodically forced, van der Pol oscillator. In: Proceedings of the ASME 2013 international mechanical engineering congress \& exposition, 1521 November 2013, San Diego, CA, IMECE2013-62465

2. Lagrange JL (1788/1965) Mecanique Analytique (2 vol). Albert Balnchard, Paris

3. van der Pol B (1920) A theory of the amplitude of free and forced triode vibrations. Radio Rev 1:701-710, 754-762

4. van der Pol B, van der Mark J (1927) Frequency demultiplication. Nature 120:363-364

5. Fatou P (1928) Suré le mouvement d'un systeme soumis á des forces a courte periode. Bull Soc Math 56:98-139

6. Krylov NM, Bogolyubov NN (1935) Methodes approchees de la mecanique non-lineaire dans leurs application a l'Aeetude de la perturbation des mouvements periodiques de divers phenomenes de resonance s'y rapportant. Academie des Sciences d'Ukraine, Kiev (in French)

7. Bogoliubov N, Mitropolsky Y (1961) Asymptotic methods in the theory of nonlinear oscillations. Gordon and Breach, New York
8. Hayashi G (1964) Nonlinear oscillations in physical systems. McGraw-Hill, New York

9. Nayfeh AH (1973) Perturbation methods. Wiley, New York

10. Nayfeh AH, Mook DT (1979) Nonlinear oscillation. Wiley, New York

11. Cap F (1974) Averaging method for the solution of nonlinear differential equations with periodic non-harmonic solutions. Int J NonLinear Mech 9:350-441

12. Rand R, Armbruster D (1987) Perturbation methods, bifurcation theory, and computer algebra. Springer, New York

13. Coppola V, Rand R (1990) Averaging using elliptic functions: approximation of limit cycle. Acta Mech 81:125-142

14. Xu Z, Cheung Y (1994) Averaging method using generalized harmonic functions for strongly nonlinear oscillators. J Sound Vib 174:563-576

15. Buonomo A (1998) On the periodic solution of the van der Pol equation for the small damping parameter. Int $\mathrm{J}$ Circuit Theory Appl 26:39-52

16. Buonomo A (1998) The periodic solution of van der Pol's equation. SIAM J Appl Math 59:156-171

17. Kovacic I, Mickens RE (2012) A generalized van der Pol type oscillator: investigation of the properties of a limit cycle. Math Comput Model 55:645-655

18. Luo ACJ (2012) Continuous dynamical systems. Higher Education Press/L\&H Scientific, Beijing/Glen Carbon

19. Luo ACJ, Huang J (2012) Analytical dynamics of period-m flows and chaos in nonlinear systems. Int J Bifurc Chaos 22(4):Article No. 1250093 
20. Luo ACJ, Huang J (2012) Analytical routines of period-1 motions to chaos in a periodically forced Duffing oscillator with twin-well potential. J Appl Nonlinear Dyn 1:73-108
21. Luo ACJ, Huang J (2012) Unstable and stable period-m motions in a twin-well potential Duffing oscillator. Discontin Nonlinearity Complex 1:113-145 